\title{
A behavior-based circuit model of how outcome expectations organize learned behavior in larval Drosophila
}

\author{
Michael Schleyer, ${ }^{1,2}$ Timo Saumweber, ${ }^{1,2}$ Wiebke Nahrendorf, ${ }^{1}$ Benjamin Fischer, ${ }^{1}$ \\ Désirée von Alpen, ${ }^{3}$ Dennis Pauls, ${ }^{3}$ Andreas Thum, ${ }^{3}$ and Bertram Gerber ${ }^{1,2,4,5,6}$ \\ ${ }^{1}$ Universität Würzburg, Biozentrum, Neurobiologie und Genetik, Am Hubland, 97074 Würzburg, Germany; ${ }^{2}$ Universität Leipzig, \\ Institut für Biologie, Genetik, Talstrasse 33, 04103 Leipzig, Germany; ${ }^{3}$ Université de Fribourg, Chemin du Musée 10, 1700 \\ Fribourg, Switzerland; ${ }^{4}$ Leibniz Institut für Neurobiologie (LIN), Abteilung Genetik von Lernen und Gedächtnis, Brenneckest. 6, 39118 \\ Magdeburg, Germany; ${ }^{5}$ Otto von Guericke Universität Magdeburg, Institut für Biologie, Verhaltensgenetik, Universitätsplatz 2, 39106 \\ Magdeburg, Germany
}

\begin{abstract}
Drosophila larvae combine a numerically simple brain, a correspondingly moderate behavioral complexity, and the availability of a rich toolbox for transgenic manipulation. This makes them attractive as a study case when trying to achieve a circuit-level understanding of behavior organization. From a series of behavioral experiments, we suggest a circuitry of chemosensory processing, odor-tastant memory trace formation, and the "decision" process to behaviorally express these memory traces-or not. The model incorporates statements about the neuronal organization of innate vs. conditioned chemosensory behavior, and the types of interaction between olfactory and gustatory pathways during the establishment as well as the behavioral expression of odor-tastant memory traces. It in particular suggests that innate olfactory behavior is responsive in nature, whereas conditioned olfactory behavior is captured better when seen as an action in pursuit of its outcome. It incorporates the available neuroanatomical and behavioral data and thus should be useful as scaffold for the ongoing investigations of the chemo-behavioral system in larval Drosophila.
\end{abstract}

[Supplemental material is available for this article.]

\begin{abstract}
Drosophila larvae, being the major feeding stages of the flies' life cycle, have a numerically simple brain, maybe 10 million times fewer neurons compared with man, and possess correspondingly moderate behavioral complexity. These features, together with the general potential of Drosophila for transgenic manipulation (Sokolowski 2001; Elliott and Brand 2008), make them an attractive study case when trying to achieve a circuit-level understanding of behavior, in particular with regard to chemosensory processing and odor-tastant learning (Gerber and Stocker 2007; Gerber et al. 2009).

Drosophila larvae innately ("innate" throughout this paper is used in the sense of experimentally naive) show positive preference for sugars (Schipanski et al. 2008) as well as to relatively low concentrations of salt (Miyakawa 1982; Niewalda et al. 2008), but negative preference for high salt concentrations (Liu et al. 2003; Niewalda et al. 2008) and for substances that taste bitter to humans (Meunier et al. 2003; Hendel et al. 2005). Regarding olfaction, larvae are typically attracted to odors but may, for some odors and at high concentrations, also show aversion (Rodrigues 1980; Cobb 1999; Boyle and Cobb 2005; Kreher et al. 2008). Given the numerical simplicity of the chemosensory system in the larva (Heimbeck et al. 1999; Ramaekers et al. 2005; Colomb et al. 2007a), a reasonably detailed understanding of innate gustatory and olfactory behavior can be reckoned with (Gerber and Stocker 2007; Kreher et al. 2008; Gerber et al. 2009) (see Discussion).
\end{abstract}

${ }^{6}$ Corresponding author.

E-mail bertram.gerber@uni-leipzig.de.

Article is online at http://www.learnmem.org/cgi/doi/10.1101//m.2163411.
To complicate matters, however, olfactory larval behavior can be flexible: Larvae can be differentially conditioned to associate one odor with a sweetened reward substrate, and another odor with an unsweetened substrate. After such training, larvae prefer the previously rewarded over the previously nonrewarded odor in a binary choice assay (Scherer et al. 2003; Neuser et al. 2005). Also, by punishing one odor with a bitter or high-concentration salt taste, larvae can be conditioned aversively to odors (Gerber and Hendel 2006; Niewalda et al. 2008).

Presently, the cellular site(s) of these kinds of learning, as well as their molecular mechanisms, are the topic of ongoing research, and one can be hopeful that a comparably detailed picture of these processes can be obtained in the larva as it has been obtained for adult Drosophila (Heisenberg 2003; Gerber et al. 2004a, 2009) (see Discussion) and bees (Menzel 2001; Giurfa 2007). However, there remains a gap in our understanding of how olfactory memory traces, once established, actually organize behavior, and how innate and learned olfactory behavior are integrated. Notably, the psychological nature of olfactory behavior as response or action is under continued debate: Within cartesian tradition, conditioned behavior often is explained by a change in value of the odor (e.g., Fiala 2007); that is, as result of appetitive training, the odor itself is something "good" for the animals and therefore they approach it. In other words, learned olfactory behavior, just as innate olfactory behavior, is regarded as a response to the odor.

Alternatively, Gerber and Hendel (2006) (see also Dickinson 2001; Elsner and Hommel 2001; Hoffmann 2003) suggested that it is more fruitful to view appetitive conditioned behavior as an action, taken in search of food: Specifically, at the moment of testing the difference between what the animals "expect" (based on 
olfactory memory) minus what they "observe" (based directly on gustatory input) can provide the animals with an estimate of their behaviors' expected gain in terms of finding food. If this expected gain is positive, i.e., if memory promises a situation better than the current one, the larva moves toward the previously reinforced odor. Thus, Gerber and Hendel (2006) suggested that it is this expected gain of food, rather than the value of the memory trace per se, or of the value of the testing situation per se, which is the immediate cause of learned behavior. In an analogous manner, Gerber and Hendel (2006) interpreted conditioned aversion as escape behavior. In this case, the expected gain assumes the form of a relief from punishment.

Here, we first ask whether innate gustatory behavior is affected by the presence of odors and whether, in turn, innate olfactory behavior is affected by the presence of tastants. Regarding associative odor-taste learning, we then ask whether and how learned olfactory behavior is affected by the presence of tastants. We report that learned, but not innate, olfactory behavior is affected by the presence of tastants, and propose a minimal, neuroanatomically plausible circuitry that can accommodate the presented behavioral as well as the available neurobiological data. We suggest that the modulating effects that the tastants can exert on learned olfactory behavior at the moment of testing ensures the organization of this learned, but not of innate, olfactory behavior according to its expected outcome.

\section{Results}

\section{Experiment 1: Is innate gustatory behavior affected by the presence of odor?}

We offer experimentally naive larvae a choice between two halves of a Petri dish: one filled with pure agarose, the other filled with agarose plus tastant (either $2 \mathrm{M}$ fructose, $5 \mathrm{mM}$ quinine, or $1.5 \mathrm{M}$ salt). Contemplating the time courses of gustatory behavior, which is positive preference with regard to 2 $\mathrm{M}$-fructose (Fig. 1A) and negative preference for $5 \mathrm{mM}$ quinine (Fig. 1B) as well as for $1.5 \mathrm{M}$ salt (Fig. 1C), we choose the 2-min time point (when gustatory behavior has not yet reached its asymptote) to test whether the presence of odor (either $n$-amylacetate diluted 1:50 in paraffin or undiluted 1-octanol) would alter gustatory behavior. This is not the case, either with regard to fructose

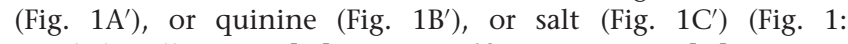
Kruskal-Wallis tests; $\left[\mathrm{A}^{\prime}\right] \mathrm{H}=1.4, d f=2, P=0.51 ;\left[\mathrm{B}^{\prime}\right] \mathrm{H}=2.9$, $d f=2, P=0.24 ;\left[C^{\prime}\right] \mathrm{H}=3.6, d f=2, P=0.16$ ). The same holds shading of the boxes.
A

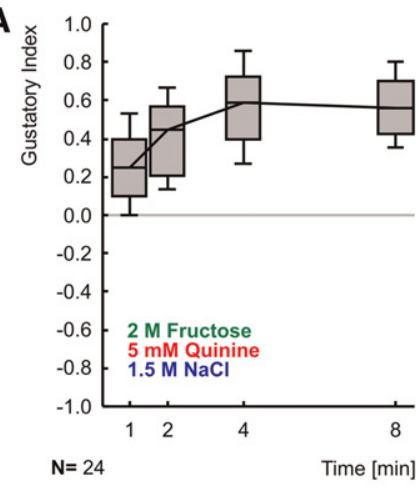

$\mathbf{A}^{\prime}$

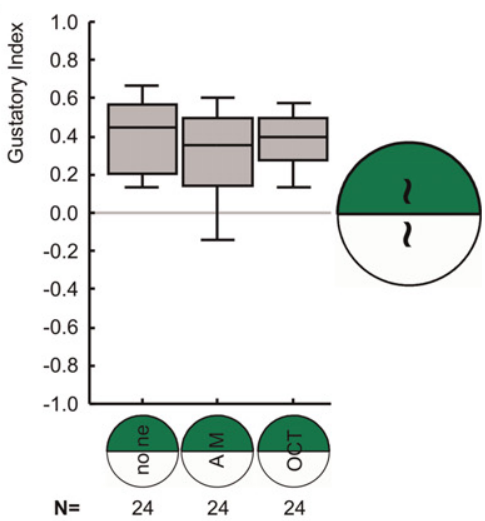

B

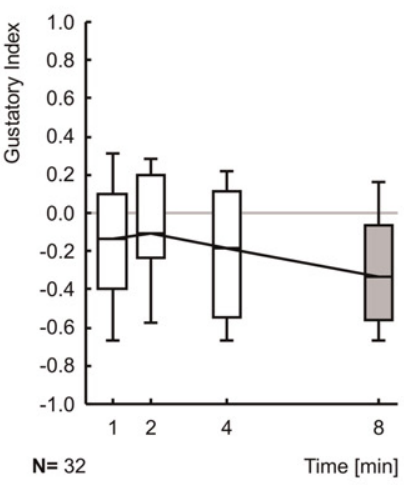

$\mathbf{B}^{\prime}$

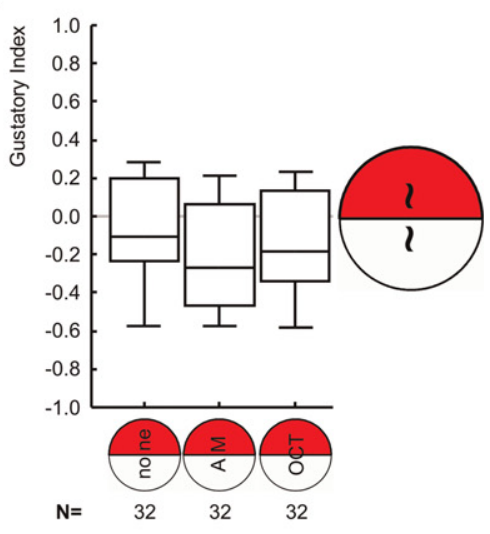

C

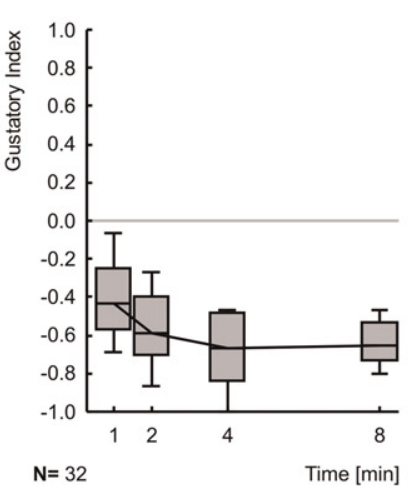

$\mathbf{C}^{\prime}$

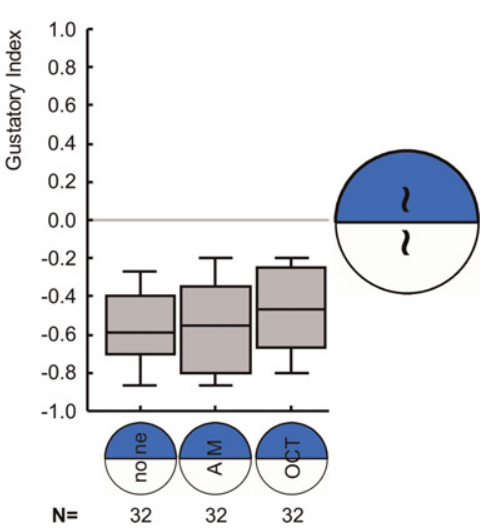

Figure 1. Is innate gustatory behavior affected by ambient odor? Displayed are the tastant preferences toward $\left(A, A^{\prime}\right) 2 \mathrm{M}$ fructose; $\left(B, B^{\prime}\right) 5 \mathrm{mM}$ quinine; and $\left(C, C^{\prime}\right) 1.5 \mathrm{M}$ salt. Larvae show positive preference toward fructose and negative preference toward quinine and salt. $A, B$, and $C$ show preferences over time; $A^{\prime}, B^{\prime}$, and $C^{\prime}$ show preference values after $2 \mathrm{~min}$, measured in the presence of either no odor, $n$-amylacetate, or 1-octanol. Please note that the "none" scores in $A^{\prime}, B^{\prime}$, and $C^{\prime}$ represent the " $2 \mathrm{~min}^{\prime \prime}$ data from $A, B$, and $C$, respectively. The box plots show the median as the bold line, $25 \%$ and $75 \%$ quantiles as the box boundaries, and $10 \%$ and $90 \%$ quantiles as whiskers. Significant differences from zero ([A,B,C]: $P<0.05 / 4,\left[A^{\prime}, B^{\prime}, C^{\prime}\right]: P<0.05 / 3$, one-sample sign tests) are indicated by

true when gustatory behavior is scored at later time points (8 min) (not shown), when tastant effects are overall stronger.

Next, following the approach of Shiraiwa (2008), we ask whether behavior toward a fructose concentration, which is just about threshold in the absence of odor (i.e., between 0.005 and $0.015 \mathrm{M}$ ) (Fig. 2A-C) can be pushed above threshold by the presence of an odor; this is not the case (Fig. 2: Kruskal-Wallis tests; $\left[\mathrm{A}^{\prime}\right] \mathrm{H}=0.77, d f=2, P=0.68 ;\left[\mathrm{B}^{\prime}\right] \mathrm{H}=2.9, d f=2, P=0.23$; 

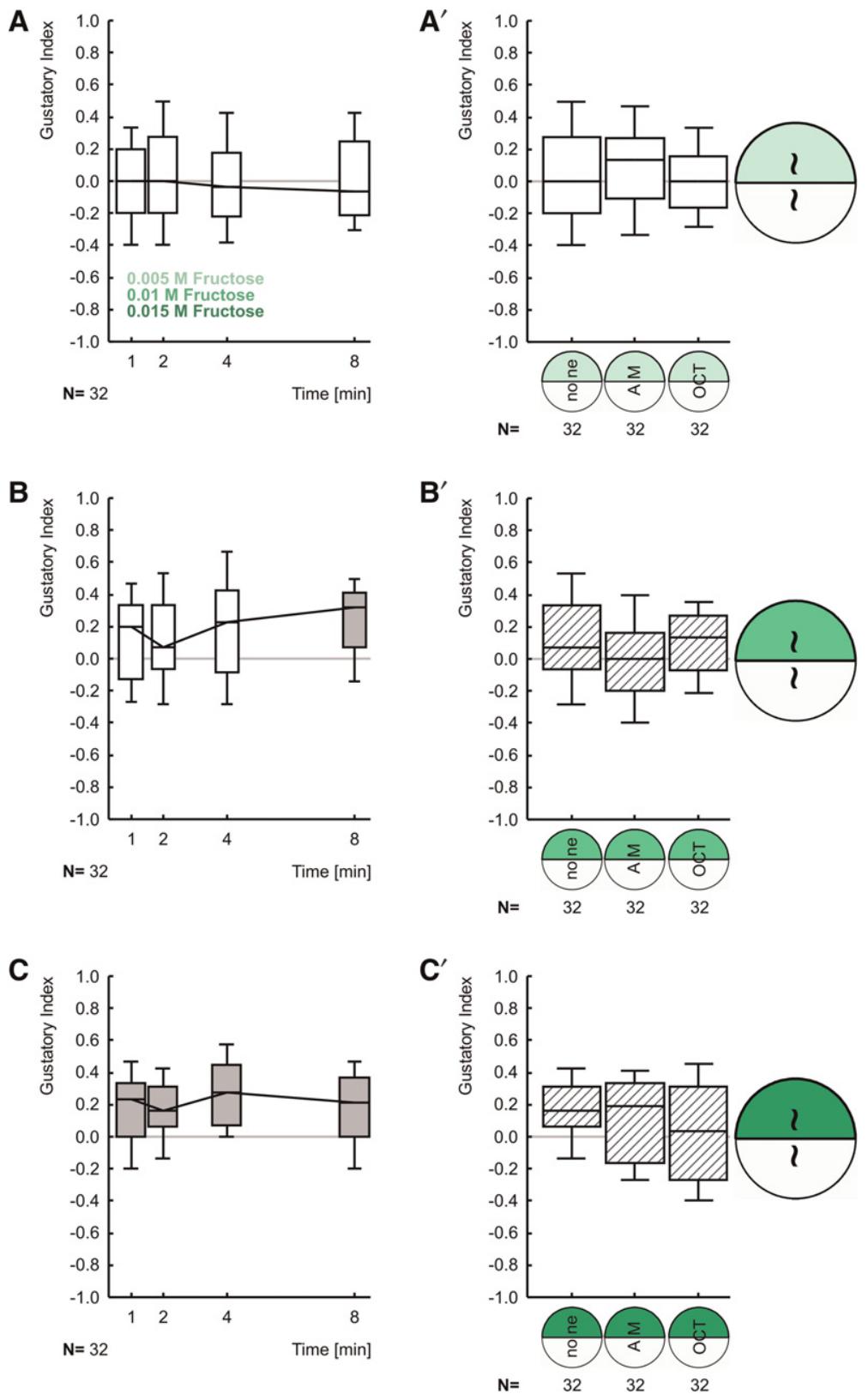

Figure 2. Do odors affect near-threshold fructose preference? Gustatory preference toward $\left(A, A^{\prime}\right)$ $0.005 \mathrm{M}$ fructose, $\left(B, B^{\prime}\right) 0.01 \mathrm{M}$ fructose, and $\left(C, C^{\prime}\right) 0.015 \mathrm{M}$ fructose. $A, B$, and $C$ show preferences over time, $A^{\prime}, B^{\prime}$, and $C^{\prime}$ show preference values after $2 \mathrm{~min}$, measured in the presence of either no odor, $n$-amylacetate, or 1-octanol. Please note that the "none" scores in $A^{\prime}, B^{\prime}$, and $C^{\prime}$ represent the

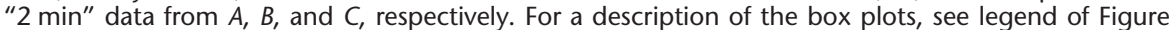
1. Significant differences from zero $(P<0.05 / 4$, one-sample sign tests) are indicated by shading of the boxes. Hatched shading of the boxes indicates significant differences from zero at least for the pooled data ( $B^{\prime}$ and $C^{\prime}, P<0.5 / 3$ in one-sample sign tests in both cases). Thus, as intended, at the chosen concentration range, fructose preference is just around threshold.

$\left.\left[\mathrm{C}^{\prime}\right]: \mathrm{H}=3.0, d f=2, P=0.22\right)$. According to the same rationale, we note that odors do not alter near-threshold behavior toward quinine (Fig. 1B's ). Regarding salt, we correspondingly seek to take advantage of the fact that behavior toward salt changes from negative preference at high salt concentration toward positive preference as concentration is decreased (Niewalda et al. 2008). The point of draw between these two behavioral tendencies is $0.25 \mathrm{M}$ (Niewalda et al. 2008), which we confirm here (Fig. 3A: one-sample sign tests; $P>0.05 / 4$ in all cases). We reasoned that at this salt concentration our assay should be most sensitive when testing for any modulation by odors: at this concentration, the positive and negative behavioral tendencies of salt just cancel out, so it should be particularly easy to "tip the balance" toward one or the other kind of behavior. Such modulating effect of odor, however, is not observed (Fig. 3B: Kruskal-Wallis test; $\mathrm{H}=0.1$, $d f=2, P=0.96$ ).

Thus, innate gustatory behavior is "insulated" against olfactory processing. Tastants therefore appear of inherent, odor-independent value to the larvae; this value is the direct basis for innate gustatory behavior.

\section{Experiment 2: Is innate olfactory behavior altered in the presence of tastants?}

We next ask whether, in turn, olfactory behavior is modulated by taste processing. Larvae are tested for their choice between an odor-filled container on one side and an empty container on the other side of a Petri dish. This test we perform on either a pure substrate, or on substrates with added fructose $(2 \mathrm{M})$, quinine $(5 \mathrm{mM})$, or salt (either $0.25 \mathrm{M}$ or $1.5 \mathrm{M})$. We find that neither for $n$-amylacetate (Fig. 4A) nor for 1-octanol (Fig. 4B) olfactory behavior is modified by the substrate condition (Fig. 4: KruskalWallis tests; [A] $\mathrm{H}=6.9, d f=4, P=$ $0.14 ;[\mathrm{B}] \mathrm{H}=4.5, d f=4, P=0.34)$, even when odors are diluted to yield only moderate levels of attraction which arguably are easier to be modulated (Fig. 4: Kruskal-Wallis tests; [C] $\mathrm{H}=0.52, d f=$ $4, P=0.97 ;[\mathrm{D}] \mathrm{H}=8.6, d f=4, P=0.1$ ). We notice a small and nonsignificant tendency of higher attraction toward $n$-amylacetate (diluted 1:50) in the presence of both fructose and quinine, compared with the values obtained on the pure, tasteless substrate (Fig. 4A); the same trend had also been found in previous experiments (data not shown). However, we see this trend neither using a different concentration of $n$-amylacetate (Fig. 4B) nor for using 1-octanol (Fig. 4C,D).

Thus, to the extent tested, innate olfactory behavior seems to be "insulated" from taste processing. This suggests that odors are of inherent value to experimentally naive larvae and that this value, independent of taste processing, is the basis for innate odor attraction. In Figure 11A (below), we graphically represent this mutual independence between smell and taste behavioral systems.

\section{Experiment 3: Expected gain drives learned olfactory behavior}

The above experiments suggest a mutual independence of innate olfactory and gustatory processing; however, associative training 
A

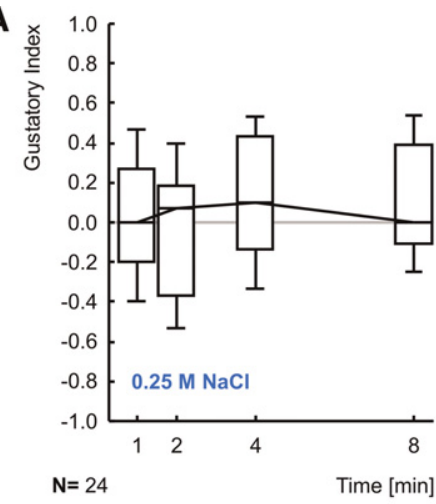

B

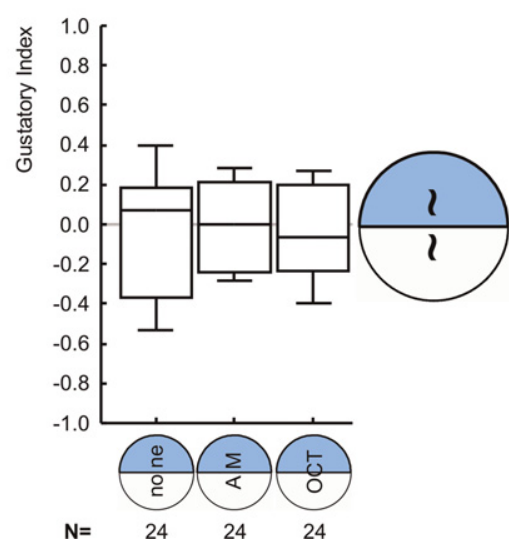

Figure 3. Do odors tip the balance between attraction and avoidance for salt preference? $(A)$ Preferences toward $0.25 \mathrm{M}$ salt in the absence of odor, measured over time. (B) Preference values after $2 \mathrm{~min}$ in the presence of either no odor, $n$-amylacetate, or 1-octanol. Please note that the

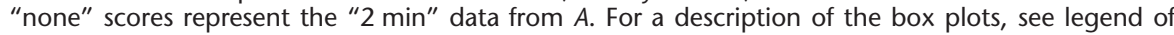
Figure 1. Values of none of the groups are significant different from zero $([A] P>0.05 / 4 ;[B] P>$ $0.05 / 3$, one-sample sign tests) arguing that as intended the attractive and aversive tendencies of salt at this concentration cancel out.

with odors and tastants can modify olfactory behavior (Scherer et al. 2003; Neuser et al. 2005; Gerber and Stocker 2007). Clearly, the formation of an odor-taste memory trace requires an interaction between olfactory processing and a taste-triggered reinforcement signal (Schwaerzel et al. 2003; Schroll et al. 2006) (Fig. 11B, below; for a discussion see Gerber et al. 2004a; 2009). The following experiments by Gerber and Hendel (2006) had been designed to see whether in addition there is a second kind of odor-taste interaction during the translation of such memory traces into conditioned behavior (Fig. 5; for the corresponding Olfactory Index values see Supplemental Fig. S2). They trained fruit fly larvae to associate an odor either with sugar, quinine, or salt (salt being used at either high, medium, or low concentration; this classification is based on the relative preference between quinine and salt (Supplemental Fig. S1: KruskalWallis test: $\mathrm{H}=178.9, d f=8, P<0.05)$. A second odor was always presented without any reinforcer. They then tested for the choice between the two odors in either the absence or presence of that reinforcer which had been used for training. If the training reinforcer was absent at test (Fig. 5A), larvae behaviorally expressed appetitive memory after sugar as well as after low-salt training; after aversive training with either quinine, high salt, or medium salt, however, animals did not express any memory (Fig. 5A: Kruskal-Wallis test; $\mathrm{H}=26.4$, $d f=4, P<0.05)$. If, in turn, the training reinforcer was present during test (Fig. 5B), the inverted pattern of results was found: larvae showed no conditioned behavior in the presence of the appetitive reinforcers, whereas they did show conditioned aversive behavior in the presence of the aversive reinforcers (Fig. 5B: Kruskal-Wallis test; $\mathrm{H}=20.9$,
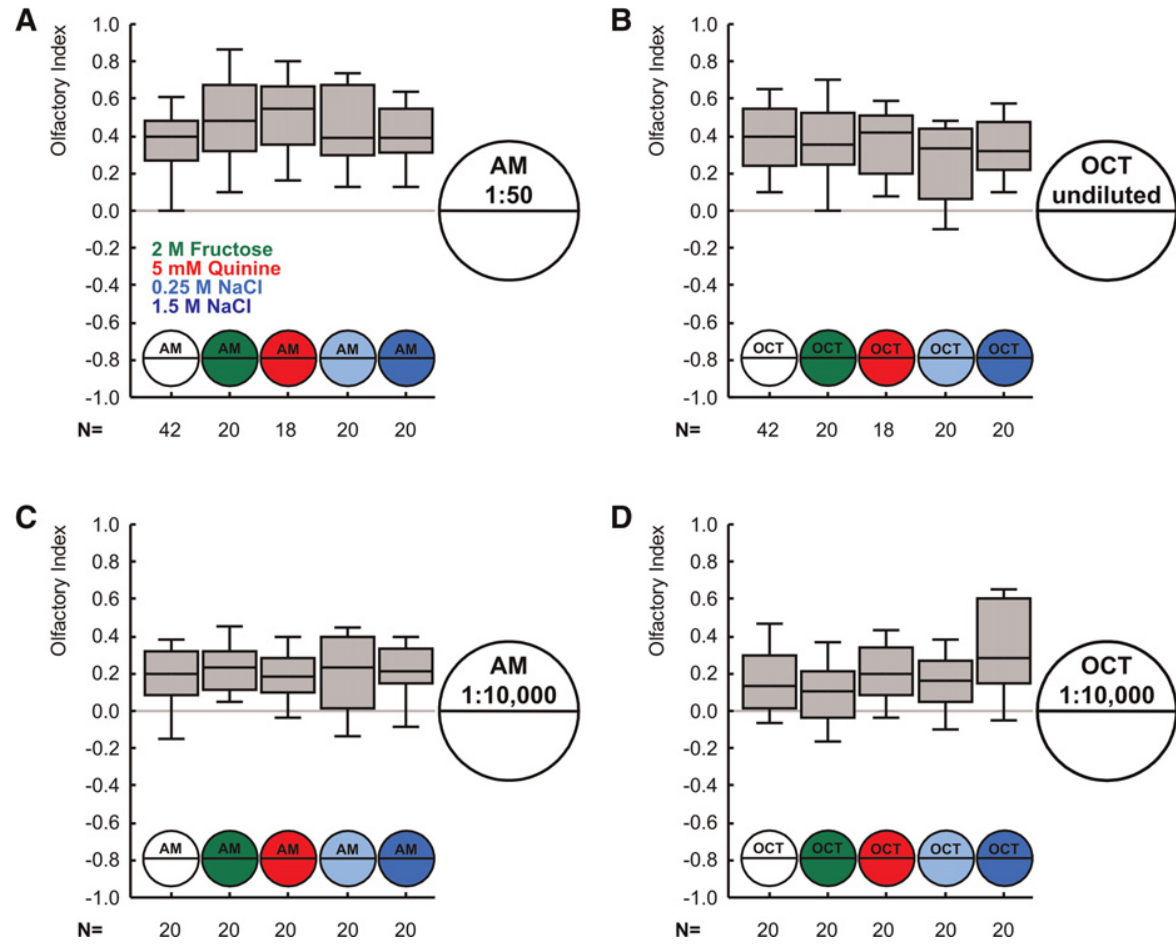

Figure 4. Is innate olfactory behavior altered in the presence of tastants? The Olfactory Index is displayed, measured on the indicated tastant-substrates, regarding $(A) n$-amylacetate diluted 1:50 in paraffin oil, $(B)$ undiluted 1-octanol, $(C) n$-amylacetate diluted 1:10,000 in paraffin oil, and $(D)$ 1-octanol diluted 1:10,000 in paraffin oil. For a description of the box plots, see legend of Figure 1. Pooled data of each graph are significantly different from zero $(P<0.05 / 4$, one-sample sign tests). 

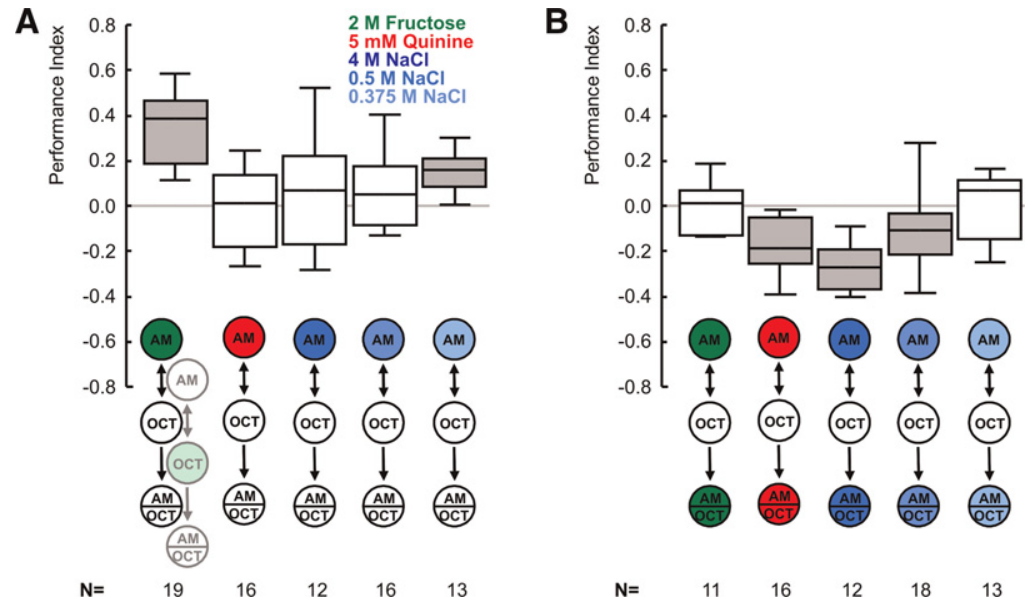

$\mathbf{N}=\quad \begin{array}{lllll}11 & 16 & 12 & 18 & 13\end{array}$

Figure 5. Expected gain drives learned olfactory behavior. Behavioral expression of associative memory, as measured by the Performance Index, in $(A)$ the absence or $(B)$ the presence of the training reinforcer. Appetitive memories are expressed only in absence, aversive memories only in presence of the training reinforcer. The sketches below the boxes show the training procedures and test conditions; colored circles represent Petri dishes containing tastant, white circles represent tasteless, pure Petri dishes. For example, in the left-most panel, the larvae receive AM with reward and OCT without reward; then, they are tested for their choice between AM and OCT. The reciprocally trained group (dimmed display) receives AM without reward, whereas OCT is rewarded. From the difference in preference between the reciprocally trained groups, the Performance Index is calculated. Positive Performance Indices indicate appetitive memory, negative values aversive memory. Note that the reciprocally trained groups were run in all cases, but with the exception of the left-most panel are omitted from the sketch for clarity. Also note that in half of the cases, the sequence of training trials is as indicated (in the left-most panel, e.g., $\mathrm{AM}+/ \mathrm{OCT}$ and $\mathrm{AM} / \mathrm{OCT}+$ ), but in the other half is reverse (e.g., $\mathrm{OCT} / \mathrm{AM}+$ and $\mathrm{OCT}+/ \mathrm{AM})$. For a description of the box plots, see legend of Figure 1. Significant differences from zero ( $P<0.05 / 5$, one-sample sign tests) are indicated by shading of the boxes. (Data adapted from Gerber and Hendel [2006], with permission from the Royal Society (C) 2006.)

Fig. S1), and hence larvae started to behaviorally express their memory in the presence of quinine (Fig. 6A). If this reasoning is correct, the pattern of results should be inverted if animals were tested in the presence of the respective salt concentrations. This indeed was found (Fig. 6B: Kruskal-Wallis test; $\mathrm{H}=11.2$, $d f=2, P<0.05$ ) (the fact that, although naive larvae are indifferent between quinine and the medium salt concentration [Supplemental Fig. S1], larvae express an avoidance of the salt-associated odor after quinine/medium salt training [Fig. 6A] may suggest that the learning assay is more sensitive to pick up differences in value between quinine and medium salt processing).

Here we seek to extend these findings to appetitive memory (Fig. 7; for the corresponding Olfactory Index values see Supplemental Fig. S4). Using the one-odor version of the learning paradigm (see Materials and Methods), four groups of larvae are trained with a medium concentration of fructose $(0.2 \mathrm{M})$ as appetitive reinforcer. The following test is performed either on a pure, tasteless substrate or on a low $(0.02 \mathrm{M})$, medium $(0.2 \mathrm{M})$, or high $(2 \mathrm{M})$ concentration of fructose (Fig. 7: Kruskal-Wallis test; $\mathrm{H}=39.1, d f=4, P<0.05)$. Larvae show conditioned behavior only when the sugar concentration at the moment of test is lower than the sugar concentration during training (one-sample sign tests; $P<0.05 / 5)$, whereas animals tested on a substrate with a sugar concentration equal to or higher than during training do not (one-sample sign tests; $P>0.05 / 5$ ). Thus, given that the four left-most groups in Figure 7 all are trained the same and consequentially will all have established the same memory trace, it is not the memory trace per se that determines the behavior of the animals.

If, in turn, animals are trained with a high concentration of fructose, but are tested in the presence of the medium sugar

\section{olfactory memory) and what they} "observe" (based directly on gustatory input) can provide them with an estimate of their behaviors' expected gain:

$$
\text { Expected Gain }=\text { Expected Value }- \text { Observed Value. }
$$

Learned olfactory behavior requires this expected gain to be positive. In other words, the behavioral expression of a memory trace involves a two-step process. First, the odor activates its memory trace. Second, in an evaluative step, a comparison is made between the value of that memory trace and the gustatory value of the testing situation. If the value of the memory trace for an odor is higher than that of the gustatory situation, i.e., if there is something to gain, the larva will track down the learnt odor. If the gustatory situation, however, already is as valuable as what the memory trace is promising, conditioned behavior remains suppressed.

Notably, Honjo and Furukubo-Tokunaga (2009), in contrast to the results of Gerber and Hendel (2006) (and also to our findings below), reported that quinine-induced aversive memory can be behaviorally expressed also in the apparent absence of quinine. The investigators, however, bathe larvae for $30 \mathrm{~min}$ in quinine solution, which despite extensive washing may induce a lingering bitter aftertaste during the test (see also Discussion).

\section{Experiment 4: Independence of appetitive and aversive memory}

We next extend the above account by an 18-group experimental design in which larvae are trained differentially using one of three kinds of training regimen:

- One odor is presented with a fructose reward and the second odor without any reinforcement. 
A

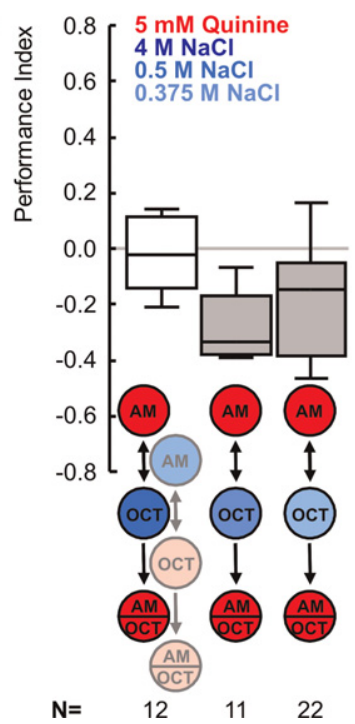

B

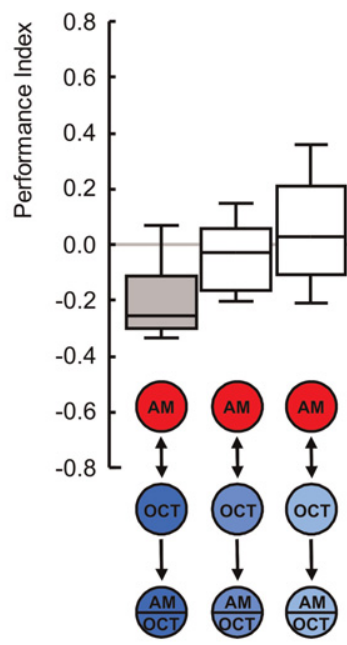

$\mathbf{N}=\quad 12 \quad 12 \quad 22$

Figure 6. Expected gain drives learned olfactory behavior. All larvae receive one odor paired with quinine, and the other odor with salt. In different groups, the concentration of salt was either high, medium, or low. Testing is performed either in the presence of quinine $(A)$ or in the presence of the salt concentration that had been used for training $(B)$. Memory expression is suppressed if none of the odors predicts a gustatory environment better than the actual test situation. Other details as in Figure 5; for a description of the box plots, see legend of Figure 1. Note that the sketches below the boxes show only one possible training regimen; the reciprocally trained group is indicated by a dimmed display in only the left-most panel of $A$. Significant differences from zero $(P<0.05 / 3$, one-sample sign tests) are indicated by shading of the boxes. (Data adapted from Gerber and Hendel [2006], with permission from the Royal Society $($ ) 2006.)

- One odor is paired with quinine punishment and the other odor without any reinforcement.

- A push-pull experimental design is used, such that one odor is rewarded and the other punished.

Animals that underwent one of these three kinds of training regimen are then tested for their choice between the trained stimuli in one of three different testing situations: on a tasteless, pure substrate, on a fructose substrate, or on a quinine substrate. Last, all experiments are performed using either the two-odor version or the one-odor version of the learning paradigm (see Materials and Methods for details) (Supplemental Fig. S5 shows the corresponding Olfactory Index scores of all groups of larvae within this experiment).

In keeping with the above account (Equation 1), scores after quinine-only training (Fig. 8A, $\mathrm{A}^{\prime}$ : Kruskal-Wallis tests; [A] $\mathrm{H}=$ 23.9, $d f=2, P<0.05$; $\left.\left[\mathrm{A}^{\prime}\right] \mathrm{H}=16.4, d f=2, P<0.05\right)$ do not reveal conditioned avoidance of the quinine-associated odor on a pure or on a sweet substrate; thus, behavior on both substrates does not differ (Fig. 8A, $\mathrm{A}^{\prime}$ : Mann-Whitney $U$-tests; $[\mathrm{A}] \mathrm{U}=97$, $P=0.52$; $\left.\left[\mathrm{A}^{\prime}\right] \mathrm{U}=103, P=0.50\right)$. This is because the "observed" pure and the "observed" fructose are both better than the "expected" quinine, such that in both cases the quinine-memory trace is not expressed in behavior. In turn, we observe conditioned avoidance in presence of quinine, different from animals' behavior on the pure substrate (Fig. 8A, $\mathrm{A}^{\prime}$ : Mann-Whitney $U$-tests; $[\mathrm{A}]$ $\left.\mathrm{U}=22, P<0.05 / 2 ;\left[\mathrm{A}^{\prime}\right] \mathrm{U}=34, P<0.05 / 2\right)$.

Scores after training with reward-only (Fig. 8B, $\mathrm{B}^{\prime}$ : Kruskal-Wallis tests; $[\mathrm{B}] \mathrm{H}=21.7, d f=2, P<0.05$; $\left[\mathrm{B}^{\prime}\right] \mathrm{H}=19.5$, $d f=2, P<0.05)$ are higher for the pure test situation than in

the presence of fructose (Fig. 8B, $\mathrm{B}^{\prime}$ : Mann-Whitney $U$-tests; [B] $\left.\mathrm{U}=14, \quad P<0.05 / 2 ; \quad\left[\mathrm{B}^{\prime}\right] \quad \mathrm{U}=34, \quad P<0.05 / 2\right)$, because the "observed" pure substrate is less valuable than the "expected" fructose, leading to the behavioral expression of the fructose-reinforced memory trace on the pure substrate. Interestingly, scores are equal in the presence and absence of quinine (Fig. 8B, $\mathrm{B}^{\prime}$ : Mann-Whitney $U$-tests; $[\mathrm{B}] \mathrm{U}=94, P=0.44$; [B'] $\mathrm{U}=124, P=$ $0.52)$, which is somewhat surprising: The above account (1) predicts that the value of quinine, if present at the moment of testing, is offset against the value of a fructose-reinforced memory trace. Thus, conditioned behavior should be expressed particularly strongly when larvae are tested in the presence of quinine after training with fructose, because the difference in value between the "observed" quinine and the "expected" fructose is particularly large. This, however, is not observed; we therefore argue that the behavioral expression of a fructose-reinforced memory trace is independent of quinine processing.

Correspondingly, one may ask whether the behavioral expression of a fructose-reinforced memory trace is possible, although the behavioral expression of a quinine-reinforced memory trace is suppressed. If this were possible, scores on a pure testing substrate (Fig. 8C, $\mathrm{C}^{\prime}$ : Kruskal-Wallis tests; $[\mathrm{C}] \mathrm{H}=25.3, d f=2$, $\left.P<0.05 ;\left[\mathrm{C}^{\prime}\right] \mathrm{H}=20.6, d f=2, P<0.05\right)$, which allows for the

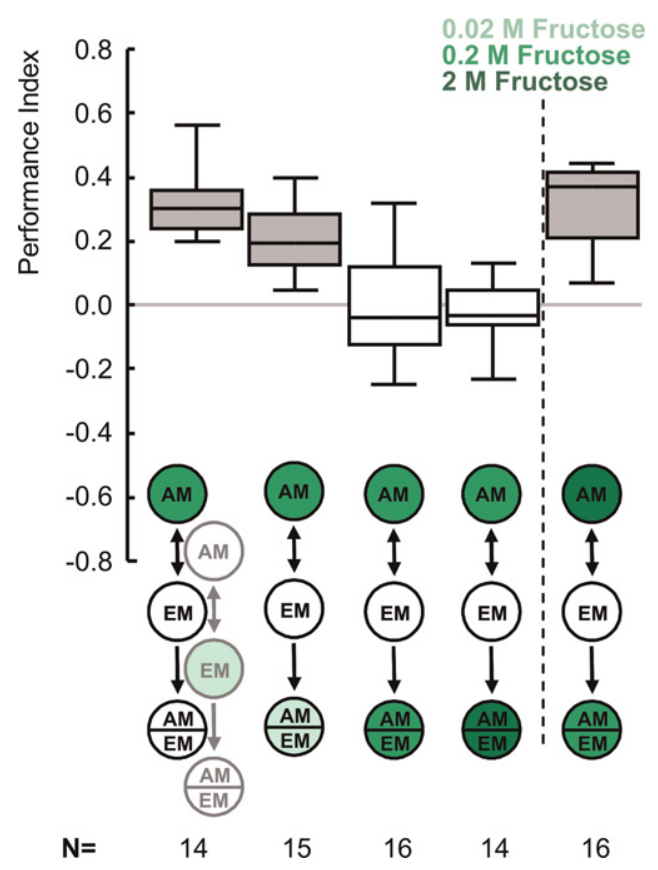

Figure 7. Expected gain drives learned olfactory behavior. Animals are trained using $n$-amylacetate (AM) and empty cups (EM). In all four leftmost panels, a medium fructose concentration $(0.2 \mathrm{M})$ is used as reinforcer during training; the subsequent test is performed either in the absence of fructose or in the presence of a lower than trained fructose concentration $(0.02 \mathrm{M})$, the medium training fructose concentration $(0.2 \mathrm{M})$, or a higher than trained fructose concentration $(2 \mathrm{M})$. In the right-most panel, a high fructose concentration ( $2 \mathrm{M})$ is used during training, but the test is performed in the presence of the medium $(0.2 \mathrm{M})$ fructose concentration. Memory is behaviorally expressed only if the fructose concentration during training is higher than the fructose concentration at the moment of test. Other details as in Figure 5; for a description of the box plots, see legend of Figure 1. Note that the sketches below the boxes show only one possible training regimen; the reciprocally trained group is indicated by a dimmed display in only the left-most panel. Significant differences from zero $(P<0.05 / 5$, one-sample sign tests) are indicated by shading of the boxes. 
A

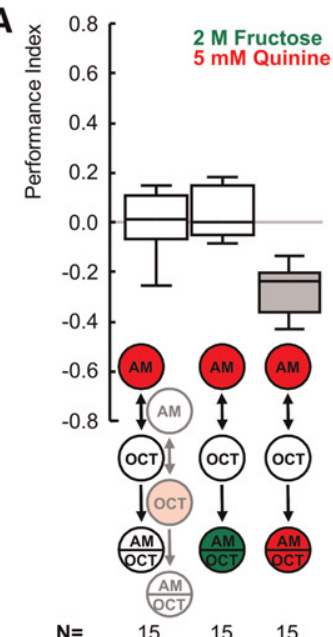

C

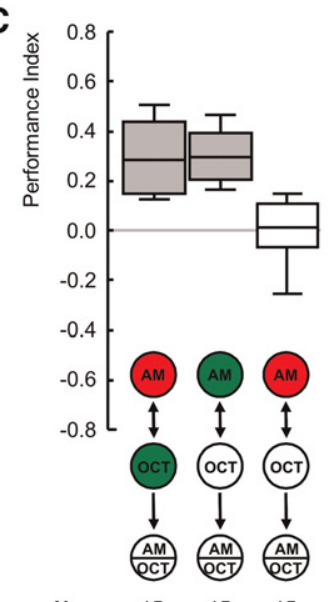

$\mathbf{N}=\quad \begin{array}{lll}15 & 15 & 15\end{array}$

E

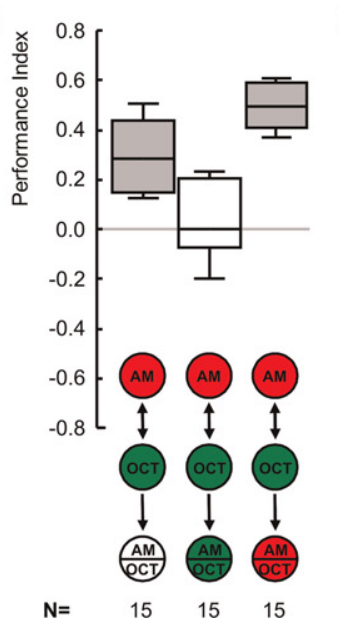

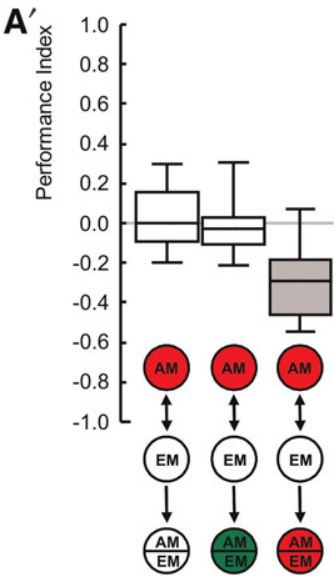

B $\times$
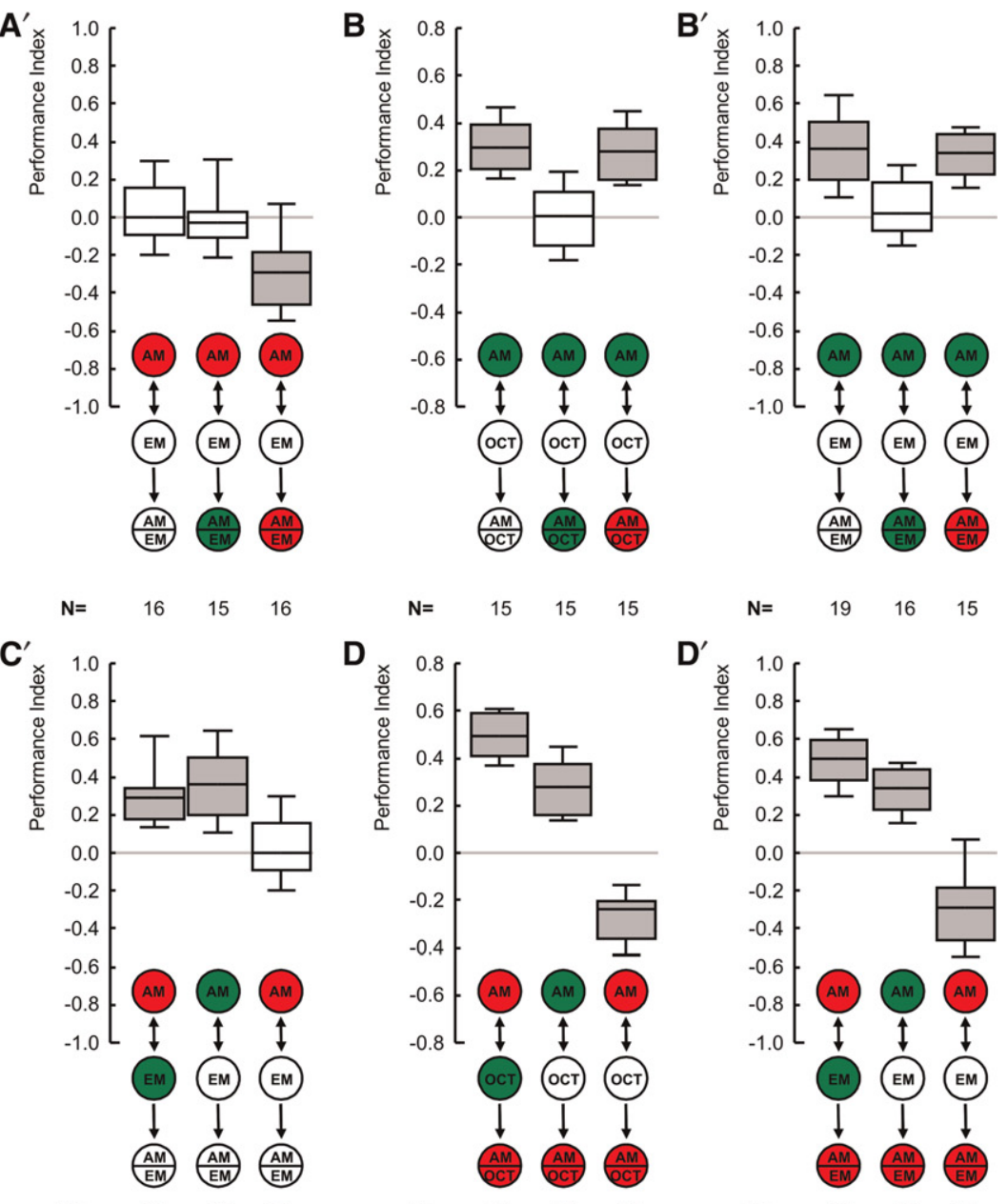

$\mathrm{N}=$

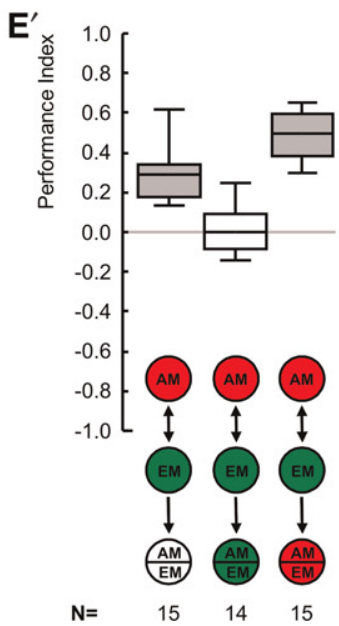

Figure 8. Independence of appetitive and aversive memory systems. $A-E$ show results of a two-odor paradigm using both $n$-amylacetae (AM) and 1-octanol (OCT), whereas $A^{\prime}, B^{\prime}, C^{\prime}, D^{\prime}$, and $E^{\prime}$ show the corresponding results of a one-odor paradigm, using only $n$-amylacetate and empty cups $(E M)$. $\left(A, A^{\prime}\right)$ After aversive-only training, larvae behaviorally express memory only in the presence of quinine. The presence of fructose has no effect. $\left(B, B^{\prime}\right)$ In contrast, after appetitive-only training, memory is behaviorally expressed only in the absence of fructose, whereas the presence of quinine has no effect. $\left(C, C^{\prime}\right)$ If animals are tested in the absence of any reinforcer, expression of aversive memories is abolished, but expression of appetitive memories remains intact. $\left(D, D^{\prime}\right)$ Animals tested on quinine show memory after all kinds of training regimen. Importantly, scores after training in a push-pull regimen using both punishment and reward are higher than those after appetitive-only training, suggesting that both an appetitive and an aversive memory are behaviorally expressed. $\left(E, E^{\prime}\right)$ After push-pull training, scores for animals tested on quinine are higher than for those tested on pure, confirming that only under these conditions, both appetitive and aversive memories are behaviorally expressed. For convenience, some data of this 18-group experiment are included in more than one graph. Other details as in Figure 5; for a description of the box plots, see legend of Figure 1. Note that the sketches below the boxes show only one possible training regimen; the reciprocally trained group is indicated by a dimmed display in only the left-most panel of $A$. Significant differences from zero $(P<0.05 / 3$, one-sample sign tests) are indicated by shading of the boxes. 
behavioral expression of a fructose-reinforced memory trace but not of a quinine-reinforced memory trace, should be equal after fructose-only and fructose-quinine training. This is indeed what we find (Fig. 8C, $C^{\prime}$ : Mann-Whitney $U$-tests; $[C] U=112$, $P=0.98 ;\left[C^{\prime}\right] \mathrm{U}=114, P=0.32$ ), suggesting that the behavioral expression of the quinine-reinforced memory trace can remain suppressed even if the fructose-reinforced memory trace is in effect.

Given these arguments for independence, we propose separate systems for steering conditioned search and conditioned escape (Fig. 11C [8,9]). Within either system, it is determined separately whether conditioned behavior is expressed or not. That is, conditioned search is expressed if the appetitive memory promises a gain in the sense of yet more reward than actually is present:

$$
\begin{aligned}
& \text { Conditioned search if: } \\
& \text { Appetitive Memory }>\text { Observed Reward. }
\end{aligned}
$$

Conditioned escape, however, remains suppressed as long as the actual situation is less bad than what aversive memory suggests, i.e., unless escape offers a gain in the sense of relief:

$$
\begin{aligned}
& \text { No conditioned escape if: } \\
& \text { Aversive Memory > Observed Punishment. }
\end{aligned}
$$

This prompted us to ask what will happen if both conditioned search and conditioned escape tendencies are activated. On a quinine substrate, which as we have shown above allows the behavioral expression of both quinine- and fructose-reinforced memory traces, we compare the scores of groups with differing histories of training (Fig. 8D,D': Kruskal-Wallis tests; [D] $\mathrm{H}=$ 34.4, $\left.d f=2, P<0.05 ;\left[\mathrm{D}^{\prime}\right] \mathrm{H}=34.1, d f=2, P<0.05\right)$. Scores in this experiment turn out to be higher after fructose-quinine training than after fructose-only training (Fig. 8D, $\mathrm{D}^{\prime}$ : Mann-Whitney $U$-tests; [D] $\mathrm{U}=32, P<0.05$; [D'] $\mathrm{U}=44, P<$ 0.05). Along the same lines, we find that after fructose-quinine training (Fig. 8E, $\mathrm{E}^{\prime}$ : Kruskal-Wallis tests; [E] $\mathrm{H}=25.4, d f=2, P<$ 0.05 ; $\left.\left[\mathrm{E}^{\prime}\right] \mathrm{H}=26.7, d f=2, P<0.05\right)$ scores are higher in the quinine than in the pure testing condition (Fig. 8E, $\mathrm{E}^{\prime}$ : Mann-Whitney $U$-tests; [E] $\left.\mathrm{U}=48, P<0.05 ;\left[\mathrm{E}^{\prime}\right] \mathrm{U}=38, P<0.05\right)$. We suggest that this is because in the presence of quinine both memory traces can be expressed behaviorally: conditioned escape from quinine is expressed because quinine is present, and conditioned search for fructose is expressed because fructose is absent. These two effects can both steer behavior independently of each other, and eventually summate in terms of the distribution of the larvae between the previously rewarded and the previously punished odor.

To further confirm our findings we partially repeat the last experiment using a high concentration sodium chloride $(1.5 \mathrm{M})$ (for further details, see legend of Fig. 9) instead of quinine (Fig. 9; for the corresponding Olfactory Index values see Supplemental Fig. S6). In replication of the results of Gerber and Hendel (2006), after punishment-only training with high-salt larvae show conditioned behavior when tested in the presence of high salt but not on a tasteless Petri dish (Fig. 9A: Mann-Whitney $U$-test; $U=3, P<0.05)$. Notably, after pushpull training with high salt and sugar (Fig. 9B), values are significantly higher when tested on high salt compared with the tasteless test condition (Fig. 9B: Mann-Whitney $U$-test; $\mathrm{U}=51, P<$ 0.05). Thus, under appropriate testing conditions fructoseinduced appetitive and salt-induced aversive memory traces can summate in behavior: on a too salty Petri dish animals both search for sugar and try to escape the high salt concentration.
A

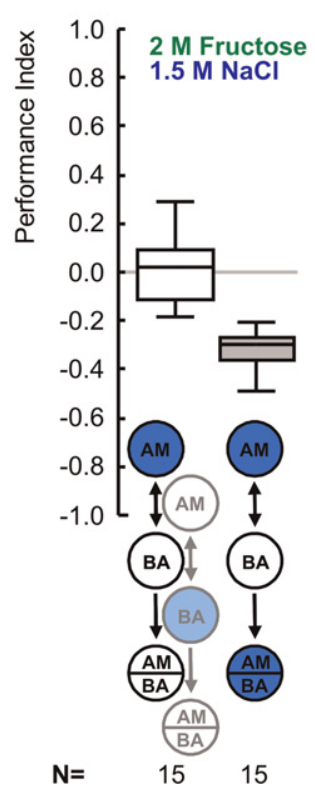

B

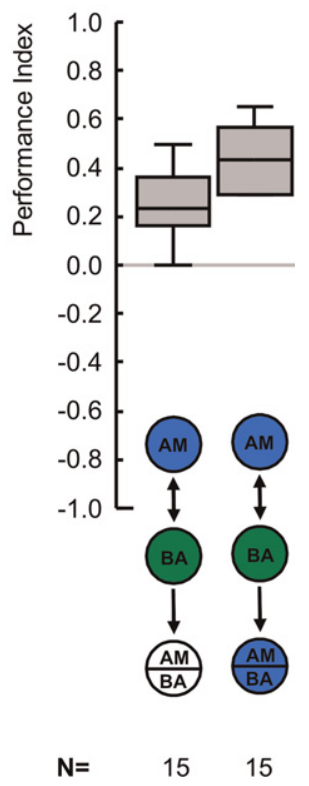

Figure 9. Independence of appetitive and aversive memory systems. The experiment partially repeats and extends the one shown in Figure 8 , using a slightly different protocol: as odors, $n$-amylacetate (AM), diluted 1:250 in paraffin, and undiluted benzaldehyde (BA) are used. Unrewarded, tasteless Petri dishes contain 2.5\% agarose, reward Petri dishes have $2 \mathrm{M}$ fructose added, and punishment Petri dishes have 1.5 $M$ sodium chloride added. $(A)$ Larvae receive aversive training with salt as punishment and are tested either in absence or presence of salt. Larvae show conditioned behavior when tested in the presence of salt but not on a tasteless Petri dish. (B) After push-pull training with salt punishment and sugar reward, performance indices are higher when tested on salt compared with the tasteless test condition, corresponding to the results of Figure 8. All other details as in Figure 5; for a description of the box plots, see legend of Figure 1. Note that the sketches below the boxes show only one possible training regimen; the reciprocally trained group is indicated by a dimmed display in only the left-most panel of $A$. Significant differences from zero $(P<0.05 / 2$, one-sample sign tests) are indicated by shading of the boxes.

\section{Discussion}

We first briefly sketch what is known neurobiologically about the establishment of smell-taste associative memory traces to provide a point of reference for our behavior-based model in the second part of this discussion.

\section{Associating smell and taste}

Larvae can learn to associate an odor with taste reinforcement. This implies convergence between olfactory and taste processing. However, no such convergence has been reported to date: the olfactory system passes on its information from the dorsal organ via only 21 olfactory sensory neurons (Heimbeck et al. 1999; Kreher et al. 2005) to the larval antennal lobe (Fig. 10A), each of them targeting just one of 21 spherical "glomerulus" compartments (Ramaekers et al. 2005). The information from a given glomerulus is conveyed further by typically just one projection neuron (Ramaekers et al. 2005; but see Marin et al. 2005), connecting to both the lateral horn, a presumed premotor center, and the mushroom bodies (Python and Stocker 2002). In the mushroom bodies' olfactory input region, the calyx, which is organized into reported 28-34 glomeruli (Marin et al. 2005; MasudaNakagawa et al. 2005, 2009; Ramaekers et al. 2005), projection neurons transmit their signal to several of a total of $\sim 600$ mature mushroom body neurons (also called Kenyon cells) (Lee et al. 
A

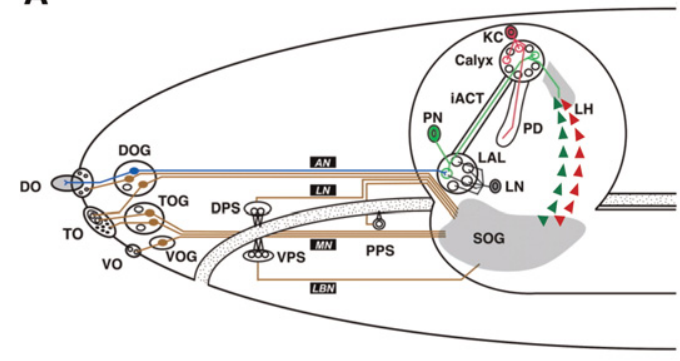

B

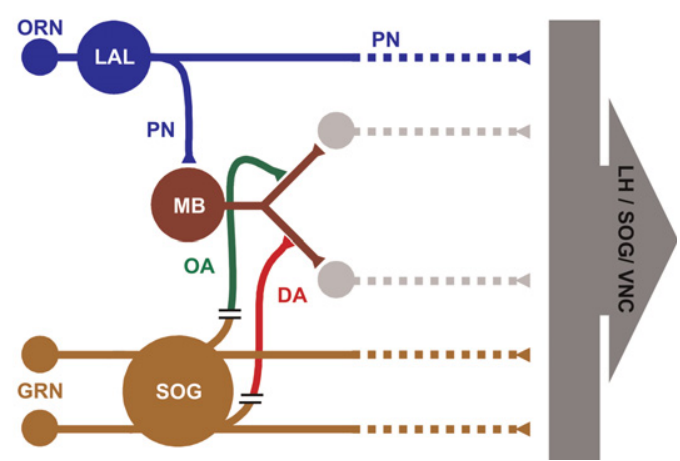

Figure 10. Neuroanatomy of the larval chemosensory system. (A) Schematic diagram of the chemosensory pathways in the larval head. (Modified from Stocker [2008], with permission from Landes Bioscience and Springer Science + Business Media (C) 2008.) (B) Simplified diagram of the chemosensory pathways in the larval brain. From the three external chemosensory organs, the dorsal organ (DO) comprises both olfactory (the "dome"; gray) and gustatory sensilla (little circles). The terminal organ (TO), the ventral organ (VO), and the dorsal, ventral, and posterior pharyngeal sense organs (DPS, VPS, PPS) include mainly taste sensilla. The cell bodies of the sensory neurons are collected in ganglia below each sense organ (DOG, TOG, VOG). Olfactory receptor neurons (ORNs, blue) project into individual glomeruli of the larval antennal lobe (LAL), which are interconnected by local interneurons (LN). Projection neurons (PNs, green) carry signals from the LAL to two higher olfactory centers, the mushroom body (MB) calyx and the lateral horn (LH). One intrinsic MB Kenyon cell $(\mathrm{KC})$ is shown in red. Gustatory receptor neurons (GRN, brown) extend to the subesophageal ganglion (SOG). Octopaminergic neurons (OA, green) are proposed to "short-circuit" a taste-driven reward signal from the SOG toward the MB; dopaminergic neurons (DA, red) carry punishment signals toward the MB. The exact neuronal elements to select particular motor programs when facing tastants and odors are unknown, but likely involve the lateral horn (LH) and ventral nerve cord (VNC).

1999; but see Technau and Heisenberg 1982). A given projection neuron innervates only one calyx glomerulus, and a given Kenyon cell collects input from between one and three (Ramaekers et al. 2005) or up to six (Masuda-Nakagawa et al. 2005) calyx glomeruli. The Kenyon cells then connect to relatively few (a reasonable guess may be between one and dozens) (Pauls et al. 2010) output neurons that have projections into the lateral horn and other potential premotor centers (Pauls et al. 2010; for the situation in adults: Ito et al. 1998; Tanaka et al. 2008; Séjourné et al. 2011). These output neurons likely receive input from many if not all mushroom body cells, thus "summing up" the total level of activation in their input section of the mushroom body.

The gustatory system, in turn, conveys taste information from three external taste organs (terminal organ, ventral organ, and the bulge of the dorsal organ) and three internal taste organs (ventral, dorsal, and posterior pharyngeal organ) (Singh and Singh 1984; Python and Stocker 2002; Gendre et al. 2004), comprising a total of $\sim 90$ gustatory sensory neurons per body side (Colomb et al. 2007a), to the subesophageal ganglion and then the ventral nerve cord (Fig. 10B; Melcher and Pankratz 2005; Colomb et al. 2007a). Thus, there is no apparent direct connection between smell and taste pathways, consistent with the lack of interaction between innate gustatory and innate olfactory behavior we report in this study.

Given this architecture, the ability of the larva to form an odor-taste associative memory trace may appear surprising. Hammer (1993) in the honeybee identified the octopaminergic $\mathrm{VUM}_{\mathrm{mx} 1}$ neuron, which likely receives gustatory input in the subesophageal ganglion and provides output to the antennal lobe, the mushroom body calyx and the lateral horn. This single, identified neuron is sufficient to mediate the rewarding function of sugar in honeybee olfactory learning (Hammer 1993) (for a description of this neuron in the fly, see Busch et al. [2009]; this neuron exists in larval Drosophila as well; A Thum, unpubl.). As in the bee, also in Drosophila there is evidence that at least some octopaminergic neurons "short-circuit" taste with smell pathways to mediate reinforcement signaling (Fig. 10B): Adult flies lacking octopamine are impaired in odor-sugar learning but not in odor-shock learning. In turn, blocking synaptic output from a subset of dopaminegic neurons impaired odor-shock learning but not odor-sugar learning (Schwaerzel et al. 2003). In larvae, the net effect of driving subsets of octopaminergic or dopaminergic neurons can substitute for reward or punishment, respectively, in olfactory learning (Schroll et al. 2006; this is not at variance with the observation that specific other subsets of these neurons serve different functions, see below). Whether and which of these neurons, in turn, are required for these two forms of learning is less clear (Honjo and Furukubo-Tokunaga 2009; Selcho et al. 2009). In any event, important for the current context is that the memory-forming convergence is not between the olfactory and the gustatory pathway itself, but between the olfactory pathway and a modulatory valuation signal ("good" or "bad," respectively). Such convergence likely happens in the mushroom bodies (Honjo and Furukubo-Tokunaga 2005, 2009; Selcho et al. 2009; Pauls et al. 2010; Michels et al. 2011; concerning adults, see Riemensperger et al. 2005; Claridge-Chang et al. 2009; reviews by Heisenberg 2003; Gerber et al. 2004a, 2009; concerning honeybees, see Hammer and Menzel 1998). If an odor is presented, a particular pattern of olfactory sensory neurons is activated, leading to the activation of a particular combination of glomeruli in the antennal lobe (Kreher et al. 2005), as well as of the projection neurons and the corresponding mushroom body neurons (Masuda-Nakagawa et al. 2005, 2009). At the same time, a tastant, e.g., sugar, activates gustatory sensory neurons that trigger the value signal ("good") via, e.g., some of the octopamine neurons (in the case of high-concentration salt or quinine: via, e.g., some of the dopaminergic neurons) and send it to many, if not all, Kenyon cells of the mushroom bodies (Honjo and Furukubo-Tokunaga 2009; Selcho et al. 2009). Conceivably, only in that subset of Kenyon cells which are activated coincidently by both the odor signal and the value signal, a memory trace is formed (Tomchik and Davis 2009; Gervasi et al. 2010; for discussion, see also Gerber et al. 2004a). The memory trace then is thought to consist of a strengthening of connection between the Kenyon cells and their output neurons: if a learned odor is presented, Kenyon cell output is strong enough to drive the output neurons for triggering conditioned behavior. Indeed, mushroom body output is a requirement for conditioned behavior (regarding adult Drosophila: Dubnau et al. 2001; McGuire et al. 2001; Schwaerzel et al. 2003). Following Selcho et al. (2009) and Aso et al. (2010), we stress that the genetic tools available at present 
to manipulate octopaminergic and dopaminergic neurons, respectively, cover anatomically and functionally heterogeneous sets of neurons. Current research is trying to identify from these sets those neurons conferring reinforcement signaling, and to tell them apart from neurons mediating other effects, e.g., regarding olfactory processing per se, gustatory processing per se, and signaling of satiety states (Honjo and Furukubo-Tokunaga 2009; Selcho et al. 2009; also see Claridge-Chang et al. 2009; Krashes et al. 2009; Aso et al. 2010 for adult Drosophila).

Regarding the below discussion, two further aspects should be noted: First, for innate olfactory behavior, the mushroom body loop is dispensable (de Belle and Heisenberg 1994), but the projection neurons are required (Heimbeck et al. 2001). This suggests that innate olfactory behavior is supported largely by the direct antennal lobe-lateral horn pathway, whereas conditioned olfactory behavior takes the indirect route via the mushroom bodies (see also Saumweber et al. 2011). Second, there is no evidence to argue that a given odor would not activate the same one subset of Kenyon cells during aversive as well as appetitive learning; this implies that appetitive and aversive memory traces for a given odor may be localized in the same Kenyon cells, but in distinct subcellular compartments (Fig. 11B, 6; see discussion in Schwaerzel et al. 2003).

\section{Integrating behavior}

Our experimental analyses of chemosensory processing focused on four kinds of behavior:

1. Innate taste behavior

2. Innate olfactory behavior

3. Conditioned olfactory behavior after appetitive learning (conditioned search)

4. Conditioned olfactory behavior after aversive learning (conditioned escape)

We asked whether the organization of these kinds of behavior is functionally independent of each other.

Clearly, both olfactory and gustatory stimuli support innate behavior. Larvae can show attraction or aversion to odors (Fig. 11A [1]; in order to make it easier to relate the behavioral evidence referred to in the text to the diagrams in the figures, we added the numerals 1-9 to the figure), and show positive or negative preference for tastants, dependent on identity and concentration of the odors and tastants, respectively (Fig. 11A [2,3]). We could not, despite effort, find any evidence of interaction between these two pathways: neither does ambient taste seem to affect olfactory attraction (Fig. 4), nor does in turn ambient odor have an effect on gustatory preference (Fig. 1). The latter may at first sight appear somewhat surprising, as Shiraiwa (2008) had found in adult Drosophila that the proboscis extension reflex, an element of feeding behavior, can be facilitated by odors. However, our results certainly do not rule out that odors may, also in the larva, have a potentiating effect on feeding behavior.

In any event, as assayed in this study, the innate locomotor tendencies supported by odors and tastants seem mutually insulated (a situation similar to what we have found for visual and olfactory processing: Yarali et al. 2006). However, the joint presentation of odor and tastant does support the establishment of an associative memory trace, clearly requiring some convergence of both kinds of signaling in the larval brain (also, the joint presentation of visual cues and tastants associatively alters visual behavior: Gerber et al. 2004b). As discussed above, this type of interaction is mediated by modulatory interneurons to "shortcircuit" taste and smell processing, employing distinct sets of neurons to signal reward and punishment (Fig. 11B [5]). As for a given
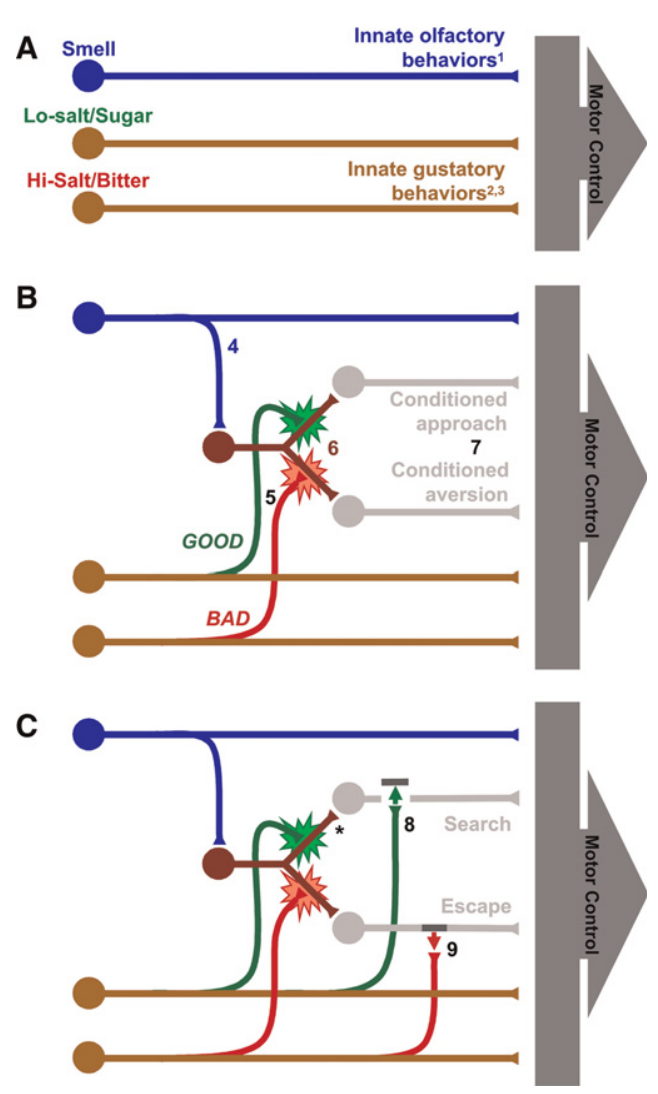

Figure 11. Behavioral-based circuit of larval chemosensory behavior. The figures illustrate, in a sequential way, which kinds of circuitry have to be proposed to accommodate the behavioral experiments reported in this study. To make it easier to relate behavioral evidence referred to in the body text to these diagrams, we added numerals 1-9. $(A)$ Innate olfactory and innate gustatory behavior are mutually "insulated" (Figs. 1,4). [1] Odors are usually attractive (Fig. 4), except at very high concentrations (e.g., Cobb and Domain 2000; Colomb et al. 2007b). [2] Larvae show negative preference in the case of high concentrations of salt and of quinine (Fig. 1B,C; Hendel et al. 2005; Niewalda et al. 2008) and positive preference in the case of low concentrated salt and sugar (Fig. 1A; Schipanski et al. 2008). [3] We present joint cellular pathways for sugar/low-concentrated salt processing and for bitter/high-concentrated salt processing, respectively, based on Hiroi et al. (2004). Separated cellular pathways for sugar/low-concentrated salt vs. bitter/high-concentrated salt are based on Marella et al. (2006). (B) Establishment of the memory trace and sketch of conditioned olfactory behavior. Larvae can associate an odor with a reward, leading to conditioned approach toward this odor (Fig. 8B; Scherer et al. 2003; Neuser et al. 2005; Schipanski et al. 2008), or with a punishment, leading to conditioned aversion to this odor (Fig. 8A; Gerber and Hendel 2006; Niewalda et al. 2008). Trivially, this requires convergence of the to-be-associated signals. [4] The bifurcation of the olfactory pathway is based on Heimbeck et al. (2001); [5] separated cellular processing of internal reward and punishment signals is based on Schwaerzel et al. (2003) and Schroll et al. (2006); [6] separated subcellular target regions of internal reward and punishment signals are based on the suggestion by Schwaerzel et al. (2003); [7] separated cellular processing of retrieval of aversive and appetitive memory traces is based on Séjourné et al. (2011). (C) Reconsidering the nature of learned behavior as conditioned search and conditioned escape behavior. Olfactory memory traces are behaviorally expressed only if animals expect to improve their situation: [8] The presence of a reward signal at the moment of testing which is at least as "good" as predicted blocks the expression of conditioned search behavior (Fig. 8B); [9] in turn, only if at the moment of testing a punishment signal is present, which is at least as "bad" as predicted, conditioned escape behavior is expressed (Fig. 8A). Please note that the suppressing effect of satiety on appetitive conditioned behavior (Krashes et al. 2009) is proposed to come about by preventing processing beyond the point marked by an asterisk in $C$ and thus likely is a process distinct from the one we investigate here. 
odor there likely is but one set of Kenyon cells available to enter into association with reward and punishment; these reward and punishment signals likely target different cellular compartments of these cells (Fig. 11B [6]), from which appetitive and aversive memory traces likely are retrieved via different sets of mushroom body-extrinsic neurons (Fig. 11B [7]).

In addition to these interactions of olfactory processing and taste-triggered reinforcement signals during training, we identify a second type of interaction. That is, whether these memory traces are behaviorally expressed or not is determined neither by the strength of the memory trace per se, nor by the circumstances of testing per se, but rather depends on a comparison between the respective memory trace and the value of the test situation: conditioned search behavior is expressed unless it is disabled by the presence of an at least as good as predicted sugar (Fig. 11C [8]). In contrast, only the presence of quinine or salt at an intensity at least as bad as predicted enables the expression of conditioned escape behavior (Fig. 11C [9]). We would like to stress that these processes require the memory trace to be "read-out" to allow for this comparison with the value of the test situation; therefore, obviously, these comparisons have to take place downstream from the site of the memory trace. This is critically different from the proposed effect of satiety: Krashes et al. (2009; loc. cit. Fig. 7) suggest that satiety prevents the very read-out of the memory trace, i.e., is acting effectively upstream of the memory trace (site labeled with * in Fig. 11C). Thus, potentially, there may be two mechanisms at operation, one regulating whether a memory trace is addressed and read-out at all (depending on satiety), and another one regulating the behavioral expression of an activated memory trace, dependent on the comparison between memory trace and the testing situation.

\section{Generality?}

As discussed above, we propose that animals express an aversive memory if they are motivated to escape from the test situation. Presenting an aversive reinforcer, as we did in our experiments, may not be the only way to induce such motivation. Indeed, there exist a broad range of studies on aversive conditioned behavior in insects that do not report the necessity of an apparent aversive reinforcer at the moment of test. Arguably, however, in these cases there may exist other sources of escape motivation:

Honjo and Furukubo-Tokunaga (2009) used a nonreciprocal quinine-learning paradigm and found aversive memory scores in the absence of quinine. However, as in that study animals were bathed in a liquid quinine solution during training, there may be a lingering bitter aftertaste at the moment of test. That is, although no quinine is presented on the test Petri dish, animals may still regard the substrate as unpleasantly bitter and seek to escape from it. Such a lingering taste may also be an explanation for the reported differences in stability of appetitive and aversive memory (half-maximal effects after 90 and $10 \mathrm{~min}$, respectively: Honjo and Furukubo-Tokunaga 2005, 2009): With the lingering taste subsiding, scores of conditioned aversion quickly decay.

Regarding larval electroshock learning, Pauls et al. (2010) report that associative aversion scores are more negative if the last training trial included electric shocks, compared with animals that received electric shock in the previous last training trial. This, again, may hint at a residual effect of electric shock that may motivate the animals' escape during test, which may be stronger for those groups that had received shock just prior to testing (in the related study by Khurana et al. [2009], data were not analyzed regarding sequence effects).

Similar arguments may apply in the case of olfactory electroshock learning in adult flies (Tully and Quinn 1985), especially as the intensity of electric shocks in this paradigm is often close to causing physical damage. Interestingly, Tempel et al. (1983) report that aversive memory scores induced by electroshocks are much less stable than appetitive memory scores induced by sugar (half-maximal effects are found after 2 and $18 \mathrm{~h}$, respectively). Again, this may hint at a carryover effect of the aversive reinforcer that vanishes after training, such that the driving force behind associative aversion may get lost. Also, before the arms of the T-maze are opened in this type of assay, about 100 fairly stressed flies are crowded in a volume approximately as small as a cherry $\left(\sim 1.5 \mathrm{~cm}^{3}\right)$, a situation that should be unpleasant to the flies. Along the same lines, the training and testing situation in sting-extension reflex conditioning of honeybees fixates the animals horizontally on their backs (Giurfa 2007, loc. cit. Fig. 1), which may not be a leisurely body posture for them.

On the other hand, after conditioning crickets with saline solution (Matsumoto and Mizunami 2002, loc. cit. Fig. 3) animals avoid the punished odor in a not apparently unpleasant situation. This observation seems to not fit to the rule of escape motivation suggested above. We note, however, that in this paradigm odor and punishment are presented not only in very close temporal but also in very close spatial proximity, potentially prompting the odor to stand in for the punishment, rather than becoming a signal for punishment. A similar argument may apply to odor-taste learning in Spodoptera littoralis larvae (Salloum et al. 2011).

Thus, we hesitate to judge whether the behavioral organization of learned behavior as found in this study is an exceptional case or whether it reveals a principle that had remained opaque in previous assays that may have "implicitly" provided a bad enough testing situation. Indeed, in a recently developed paradigm of association between odor and mechanosensory disturbance as punishment in larval Drosophila, learned behavior likewise is only revealed in the presence of that punishment (Eschbach et al. 2011). Also, Schnaitmann and colleagues, analyzing visual learning in adult flies, report that after punishing animals with formic acid during training, aversive memory is behaviorally expressed in the presence but not the absence of formic acid (Schnaitmann et al. 2010, loc. cit. Fig. 10). Importantly, in this paradigm 50-100 flies can freely move about a large, $9-\mathrm{cm}$ diameter test arena, such that there may not be any reason to escape unless "explicitly" provided by the experimenter by adding formic acid to the test situation. Similarly, flies trained to associate visual landmarks with a comfortably cool spot in an otherwise uncomfortably hot arena search at the trained location if the testing arena is uniformly hot (Ofstad et al. 2011), but not nearly as well when it is uniformly cool (M Reiser, HHMI JFRC, pers. comm.).

\section{Outlook}

Contemplating the neuronal architecture of the insect olfactory system (Fig. 10; for a recent review, see Galizia and Rössler 2010), one of the striking features is that antennal lobe output has two target areas: the mushroom bodies and the lateral horn. These, we propose from our behavior analysis (Fig. 11), correspond to one flexible, open processing stream, and one more rigid, closed one:

- Along the mushroom body route, olfactory processing is integrated with at least two kinds of gustatory signal, namely, a reinforcement signal to induce associative plasticity in the mushroom bodies, and a value signal regarding the current status of the gustatory environment. When encountering a conditioned odor, this "triadic" architecture accommodates a regulatory step, an element of "pondering" if you will, between the activated memory trace and behavior control: It integrates 
the past experience of the larva (in the form of the memory trace activated by the odor), its present matter of concern (in terms of the present gustatory environment), and its options for future action (in terms of the premotor neurons for conditioned behavior). This endows the animal with the option to express conditioned olfactory behavior or not. The flexibility and openness of this architecture, we suggest, is a basic feature of behavior organization, reflecting the fundamental uncertainty in the world as we find it in general, and the uncertainty of what will be the best action under any given set of circumstances in particular.

- In contrast, the direct antennal lobe-lateral horn pathway is relatively rigid and closed: It is effectively "insulated" against gustatory processing (as well as against visual processing: Yarali et al. 2006). There are few if any degrees of freedom along this processing stream, such that a given olfactory stimulus is, without much reference to what goes on in the "rest" of the brain, able to organize behavior. Such relatively hard-wired organization, we argue, reflects the outcome of evolutionary trial and error, a phylogenetic curbing of the initially open and flexible organization of behavior to those few odor-behavior relationships that fit under almost all circumstances.

Considering the contrast to the relatively rigid, closed processing stream along the antennal lobe-lateral horn pathway, we suggest that conditioned olfactory behavior organized along the mushroom body loop assumes characteristics of a "decision" (Zhang et al. 2007), in our case in the sense that conditioned behavior can be expressed or not. Such a "decision" appears simple enough to allow experimental access and complex enough to remain interesting.

For now, the proposed functional circuitry is merely a working hypothesis, a scaffold to investigate the cellular sites of associative plasticity, the tastant signals modulating, as well as the downstream motor effectuators organizing learned olfactory behavior. It should thus, we hope, bring us closer to a comprehensive understanding of what makes a larva do what a larva's got to do and to the implementation of this understanding into a bioinspired robot.

\section{Materials and Methods}

\section{General}

Drosophila melanogaster of the Canton-S wild-type strain are used and kept in mass culture, maintained at $25^{\circ} \mathrm{C}, 60 \%-70 \%$ relative humidity, and a $14 / 10 \mathrm{~h}$ light/dark cycle. Experiments are performed under a fume hood at $20^{\circ} \mathrm{C}$ to $24^{\circ} \mathrm{C}$ room temperature and use 5-d-old feeding-stage larvae collected from the food slurry and gently washed in tap water before the start of the experiments. Petri dishes used (Sarstedt) are of $85-\mathrm{mm}$ diameter (except in the case of the experiments displayed in Figs. 1-3, which use 52-mm Petri dishes); they are prepared freshly the day before experiments and contain solidified 1\% agarose (electrophoresis grade; Roth) (only the experiment displayed in Fig. 9 uses $2.5 \%$ agarose). As the respective experiments require, tastants (fructose [FRU; CAS: 57-48-7; purity 99\%; Roth]; quinine hemisulfate [QUI; CAS: 6119-70-6; Sigma-Aldrich], or sodium chloride [NaCl; CAS: 7647-14-5; purity 99.5\%; Roth]) are added to the agarose at the respectively indicated concentrations to create sweet, bitter, or salty substrates. Odors ( $n$-amylacetate [AM; CAS: 628-63-7; Merck]; 1-octanol [OCT; CAS: 111-87-5; Sigma-Aldrich]; benzaldehyde [BA; CAS: 100-52-7; Fluka]) are presented by custom-made Teflon containers with 5-mm diameter, covered by a lid with seven $0.5-\mathrm{mm}$ holes as soon as $10 \mu \mathrm{L}$ of odor has been loaded; dilutions are made in paraffin oil (CAS: 8012-95-1; SigmaAldrich). Only the experiments displayed in Figures 1-3 use another way of odor application (see below).
Is innate gustatory behavior affected by ambient odor?

For assaying innate gustatory behavior, 52-mm diameter Petri dishes are divided into two halves by using a vertical barrier cut from overhead transparencies (Hendel et al. 2005). One half of the dish is filled with only solidified $1 \%$ agarose and the other with agarose in addition containing one of three tastants (FRU, QUI, NaCl). Shortly before the substances solidify, the barrier is removed and Petri dishes stored for use on the following day.

Larvae are put in the middle of such a split Petri dish. After 1, 2,4 , and 8 min their location is repeatedly determined as either on the tastant side (\# $\#_{\text {TASTANT }}$ ), on the agarose-only side (\# $\#_{\text {PURE }}$ ) or in an $\sim 1$-cm-wide "middle" stripe of the plate (\# MIDDLE; for clarity, this middle stripe is not displayed in the sketches of the figures) as well as the total number of larvae \# $_{\text {TASTANT }}+\#_{\text {PURE }}+$ $\left.\#_{\text {MIDDLE }}=\#_{\text {TOTAL }}\right)$. Given that for these experiments the smalldiameter Petri dishes are used, and that we repeatedly score larval behavior at fairly short intervals, only 15 larvae are used in all gustatory behavior assays. From these data, a Gustatory Index (GI) $[-1 ; 1]$ is calculated as:

$$
\mathrm{GI}=\frac{\left(\#_{\text {TASTANT }}-\#_{\text {PURE }}\right)}{\#_{\text {TOTAL }}} .
$$

Thus, positive values indicate positive preference to the tastant, negative values negative preferences to the tastant.

To test whether innate gustatory behavior is altered by the presence of an odor, the Gustatory Index is determined (1) in the absence of odor, (2) in the presence of $n$-amylacetate (AM; diluted 1:50), or (3) in the presence of 1-octanol (OCT; undiluted).

To prevent an accumulation of the larvae at and immediately around the odor containers, in only this experimental series no odor containers are used. Rather, two $0.5-\mathrm{cm} \times 0.5-\mathrm{cm}$ filter papers are attached to the inner side of the lid of the Petri dish, each on one side of the plate, using double faced adhesive tape. Filter papers are loaded either both with $5 \mu \mathrm{L}$ of AM or both with $5 \mu \mathrm{L}$ of OCT. As control condition, no odor is added to the filter papers.

\section{Is innate olfactory behavior altered in the presence of tastants?}

To test whether, in turn, innate olfactory behavior is influenced by the presence of tastants, behavior of experimentally naive larvae toward odor is assayed on a Petri dish containing either (1) agarose-only or on Petri dishes which in addition contain (2) fructose $(2 \mathrm{M})$, (3) quinine (5 mM), (4) low salt $(0.25 \mathrm{M})$, or (5) high salt $(1.5 \mathrm{M})$.

Petri dishes (85-mm diameter) are filled with either $1 \%$ solidified agarose or with agarose plus an added tastant and are used the following day. Two Teflon containers are placed at the circumference of the Petri dish, on opposing sides; one is loaded with $10 \mu \mathrm{L}$ of odor (either AM or OCT, at the respectively indicated dilution), while the other container serves as control with no odor added (empty, EM). For all olfactory behavior assays, the large-diameter Petri dishes are used to yield sufficient distances for odor gradients to form. As in addition no temporal resolution of a larval behavior is attempted, all olfactory behavior experiments, including all learning experiments, use groups of 30 larvae. Larvae are transferred to the middle of a Petri dish; after $3 \mathrm{~min}$, we determine the number of animals at the odor side $\left(\#_{O D O R}\right)$, the number at the no-odor side $\left(\#_{\mathrm{EM}}\right)$ as well as in a 1-cm-wide middle stripe (\# $\#_{\text {MIDDLE}}$; for clarity, this middle stripe is not displayed in the sketches of the figures), and the total ( $\#_{\mathrm{ODOR}}+\#_{\mathrm{EM}}+\#_{\text {MIDDLE }}=$ $\#_{\text {TOTAL }}$ ) number of larvae and calculate an Olfactory Index (OI) $[-1 ; 1]$ as:

$$
\mathrm{OI}=\frac{\left(\#_{\mathrm{ODOR}}-\#_{\mathrm{EM}}\right)}{\#_{\mathrm{TOTAL}}} .
$$

Thus, positive values indicate attraction to the odor, negative values aversion. 


\section{Conditioned olfactory behavior: Two-odor paradigm}

Three kinds of training are used: (1) appetitive training, (2) aversive training, and (3) a "push-pull" combination using both reward and punishment. For appetitive training, larvae receive either of two training protocols: Either AM is presented with reward and OCT without reward $(\mathrm{AM}+/ \mathrm{OCT})$, or they are trained reciprocally $(\mathrm{AM} / \mathrm{OCT}+)$. For aversive training, the procedure is analogous (AM - /OCT or AM/OCT - ). For the push-pull experimental design, one odor is rewarded and another odor is punished $(\mathrm{AM}+/ \mathrm{OCT}-$ or $\mathrm{AM}-/ \mathrm{OCT}+)$. In all cases, we measure the choice between AM vs. OCT in a final test. As reward and punishment, respectively, we use fructose, quinine, or salt added to agarose, in the concentrations mentioned in the Results section.

Specifically, two odor containers are loaded with odor (unless mentioned otherwise, either with AM diluted 1:50 in paraffin oil, or with undiluted OCT) (diluting AM ensures that innate responses to AM and OCT are about equally strong [cf. Fig. 4A and $\mathrm{B}]$ ) and placed onto a Petri dish that either does or does not contain a tastant reinforcer. These two containers (both loaded with the same odor) are placed at the outer circumference of a Petri dish, on opposing sides. For the first training trial, larvae are transferred to the Petri dish; after 5 min they are transferred to a fresh dish with the alternative odor-substrate combination for the second training trial. For example, during appetitive training larvae are first exposed to $\mathrm{AM}$ in the presence of fructose $(\mathrm{AM}+)$, and then to OCT in the absence of fructose (OCT) (AM+/OCT training). This training cycle is repeated three times. Then, animals are placed in the middle of a Petri dish with AM on one side and OCT on the other. This test plate may or may not contain a tastant reinforcer, as is mentioned along the Results section.

After $3 \mathrm{~min}$, we determine the number of animals at the AM side $\left(\#_{\mathrm{AM}}\right)$, the number at the OCT side $\left(\#_{\mathrm{OCT}}\right)$, the number of larvae on the middle stripe ( $\left.\#_{\text {MIDDLE}}\right)$ and the total $\left(\#_{\mathrm{AM}}+\#_{\mathrm{OCT}}+\right.$ $\#_{\text {MIDDLE }}=\#_{\text {TOTAL }}$ ) number of larvae and calculate an Olfactory Index (OI) $[-1 ; 1]$ as:

$$
\mathrm{OI}=\frac{\left(\#_{\mathrm{AM}}-\#_{\mathrm{OCT}}\right)}{\#_{\mathrm{TOTAL}}}
$$

Then, a second group of larvae is trained reciprocally, such that OCT is rewarded and AM is not (AM/OCT +). From these alternately run, reciprocally trained groups we calculate a Performance Index (PI) $[-1 ; 1]$ as:

$$
\mathrm{PI}=\frac{\left(\mathrm{OI}_{\mathrm{AM}+/ \mathrm{OCT}}-\mathrm{OI}_{\mathrm{AM} / \mathrm{OCT}+}\right)}{2} .
$$

Thus, positive PIs indicate appetitive, negative values aversive conditioned behavior.

In half of the cases the sequence of training trials is as indicated in the previous example (i.e., $\mathrm{AM}+/ \mathrm{OCT}$ and in the reciprocal group AM/OCT + ), and in the other half of the cases the sequences are reversed (i.e., OCT/AM+ and in the reciprocal OCT $+/ A M)$. Notably, the sequence of training trials does not have an effect on behavior at test (Schleyer 2009; Saumweber et al. 2011), and hence, the assignment of data for the calculation of the PI is unproblematic (see also Appendix of Hendel et al. 2005). For aversive training and push-pull training, training is performed in an analogous way and the Performance Indices are calculated respectively as:

$$
\mathrm{PI}=\frac{\left(\mathrm{OI}_{\mathrm{AM}-/ \mathrm{OCT}}-\mathrm{OI}_{\mathrm{AM} / \mathrm{OCT}-}\right)}{2} \text { after aversive training. }
$$

$$
\mathrm{PI}=\frac{\left(\mathrm{OI}_{\mathrm{AM}+/ \mathrm{OCT}--}-\mathrm{OI}_{\mathrm{AM}-/ \mathrm{OCT}+}\right)}{2} \text { after push }- \text { pull training. }
$$

\section{Conditioned olfactory behavior: One-odor paradigm}

In order to confirm our findings we repeat the kinds of experiment described above in a number of cases, but using only one odor.
That is, training and test are run in the very same way as described, but OCT is omitted throughout, such that instead of loading the respective container with OCT, an empty container (EM) is used. Thus, appetitive training follows the logical structure of training as either $\mathrm{AM}+/ \mathrm{EM}$ or in the reciprocal $\mathrm{AM} / \mathrm{EM}+$. Aversive training is run as either $\mathrm{AM}-/ \mathrm{EM}$ or in the reciprocal as $\mathrm{AM} / \mathrm{EM}-$, and the push-pull version uses either $\mathrm{AM}+$ / $\mathrm{EM}-$ or $\mathrm{AM}-/ \mathrm{EM}+$ training. Again, the sequence of trial types is reversed in half of the cases (see above). Then, larvae are tested for their choice between AM and EM on the respectively mentioned type of substrate and data are analyzed as detailed above.

\section{Data analysis}

Given that behavioral data typically are not normally distributed (and, in particular, as data within restricted intervals by definition are not normally distributed), nonparametric statistics (onesample sign test, Kruskal-Wallis test, Mann-Whitney $U$-test) are applied throughout, using Statistica 7.1 (StatSoft) for the PC (the one-sample sign test uses a web-based statistic tool provided on http://www.fon.hum.uva.nl/Service/Statistics.html). When multiple one-sample or pair-wise comparisons are made within an experiment, a Bonferroni correction keeps the experimentwide error rate $<5 \%$ by dividing the critical $P$-value by the number of tests (e.g., for three tests $P<0.05 / 3$ ); this is a conservative approach to significance testing. Data are displayed as box plots, where the middle line shows the median, the box boundaries the $25,75 \%$ quantiles, and the whiskers the $10,90 \%$ quantiles.

\section{Acknowledgments}

This work was supported by the BMBF Bernstein Focus Program Insect-Inspired Robotics and a Heisenberg Fellowship of the DFG (to B.G.). M.S. was supported by a PhD fellowship of the Studienstiftung des deutschen Volkes; T.S. was supported by a $\mathrm{PhD}$ fellowship of the Excellence Initiative of the German Federal and State Governments to the Graduate School of Life Sciences (GSLS), University of Würzburg; and D.v.A., D.P., and A.T. were supported by the Swiss National Funds (3100A0105517).

Experimental contributions of S. Ehser, K. Gerber, K. Karg, A. Kronhard, and K. Tschirner, as well as discussions with M. Heisenberg, T. Hendel, J. Hoffmann, D. Planitzer, W. Rössler, and A. Yarali are gratefully acknowledged.

\section{References}

Aso Y, Siwanowicz I, Bracker L, Ito K, Kitamoto T, Tanimoto H. 2010. Specific dopaminergic neurons for the formation of labile aversive memory. Curr Biol 20: $1445-1451$.

Boyle J, Cobb M. 2005. Olfactory coding in Drosophila larvae investigated by cross-adaptation. J Exp Biol 208: 3483-3491.

Busch S, Selcho M, Ito K, Tanimoto H. 2009. A map of octopaminergic neurons in the Drosophila brain. J Comp Neurol 513: 643-667.

Claridge-Chang A, Roorda RD, Vrontou E, Sjulson L, Li H, Hirsh J, Miesenbock G. 2009. Writing memories with light-addressable reinforcement circuitry. Cell 139: 405-415.

Cobb M. 1999. What and how do maggots smell? Biol Rev 74: 425-459.

Cobb M, Domain I. 2000. Olfactory coding in a simple system: Adaptation in Drosophila larvae. Proc Biol Sci 267: 2119-2125.

Colomb J, Grillenzoni N, Ramaekers A, Stocker RF. 2007a. Architecture of the primary taste center of Drosophila melanogaster larvae. J Comp Neurol 502: $834-847$

Colomb J, Grillenzoni N, Stocker RF, Ramaekers A. 2007b. Complex behavioural changes after odour exposure in Drosophila larvae. Anim Behav 73: 587-594.

de Belle JS, Heisenberg M. 1994. Associative odor learning in Drosophila abolished by chemical ablation of mushroom bodies. Science 263: 692-695.

Dickinson A. 2001. The 28th Bartlett Memorial Lecture. Causal learning: An associative analysis. Q J Exp Psychol 54: 3-25.

Dubnau J, Grady L, Kitamoto T, Tully T. 2001. Disruption of neurotransmission in Drosophila mushroom body blocks retrieval but not acquisition of memory. Nature 411: 476-480.

Elliott DA, Brand AH. 2008. The GAL4 system: A versatile system for the expression of genes. Methods Mol Biol 420: 79-95. 
Elsner B, Hommel B. 2001. Effect anticipation and action control. J Exp Psychol 27: 229-240.

Eschbach C, Cano C, Haberkern H, Schraut K, Guan C, Triphan T, Gerber B. 2011. Associative learning between odorants and mechanosensory punishment in larval Drosophila. J Exp Biol (in press).

Fiala A. 2007. Olfaction and olfactory learning in Drosophila: Recent progress. Curr Opin Neurobiol 17: 720-726.

Galizia CG, Rössler W. 2010. Parallel olfactory systems in insects: Anatomy and function. Anпu Rev Entomol 55: 399-420.

Gendre N, Luer K, Friche S, Grillenzoni N, Ramaekers A, Technau GM, Stocker RF. 2004. Integration of complex larval chemosensory organs into the adult nervous system of Drosophila. Development 131: 83-92.

Gerber B, Hendel T. 2006. Outcome expectations drive learned behaviour in larval Drosophila. Proc R Soc B 273: 2965-2968.

Gerber B, Stocker RF. 2007. The Drosophila larva as a model for studying chemosensation and chemosensory learning: A review. Chem Senses 32: $65-89$.

Gerber B, Tanimoto H, Heisenberg M. 2004a. An engram found? Evaluating the evidence from fruit flies. Curr Opin Neurobiol 14: 737-744.

Gerber B, Scherer S, Neuser K, Michels B, Hendel T, Stocker RF, Heisenberg M. 2004b. Visual learning in individually assayed Drosophila larvae. J Exp Biol 207: 179-188.

Gerber B, Stocker RF, Tanimura T, Thum AS. 2009. Smelling, tasting, learning: Drosophila as a study case. Results Probl Cell Differ 47: $139-185$.

Gervasi N, Tchenio P, Preat T. 2010. PKA dynamics in a Drosophila learning center: Coincidence detection by rutabaga adenylyl cyclase and spatial regulation by dunce phosphodiesterase. Neuron 65: 516-529.

Giurfa M. 2007. Behavioral and neural analysis of associative learning in the honeybee: A taste from the magic well. J Comp Physiol A Neuroethol Sens Neural Behav Physiol 193: 801-824.

Hammer M. 1993. An identified neuron mediates the unconditioned stimulus in associative olfactory learning in honeybees. Nature 366: $59-63$.

Hammer M, Menzel R. 1998. Multiple sites of associative odor learning as revealed by local brain microinjections of octopamine in honeybees. Learn Mem 5: 146-156.

Heimbeck G, Bugnon V, Gendre N, Haberlin C, Stocker RF. 1999. Smell and taste perception in Drosophila melanogaster larva: Toxin expression studies in chemosensory neurons. J Neurosci 19: 6599-6609.

Heimbeck G, Bugnon V, Gendre N, Keller A, Stocker RF. 2001. A central neural circuit for experience-independent olfactory and courtship behavior in Drosophila melanogaster. Proc Natl Acad Sci 98: 15336-15341.

Heisenberg M. 2003. Mushroom body memoir: From maps to models. Nat Rev Neurosci 4: 266-275.

Hendel T, Michels B, Neuser K, Schipanski A, Kaun K, Sokolowski MB, Marohn F, Michel R, Heisenberg M, Gerber B. 2005. The carrot, not the stick: Appetitive rather than aversive gustatory stimuli support associative olfactory learning in individually assayed Drosophila larvae. J Comp Physiol A Neuroethol Sens Neural Behav Physiol 191: 265-279.

Hiroi M, Meunier N, Marion-Poll F, Tanimura T. 2004. Two antagonistic gustatory receptor neurons responding to sweet-salty and bitter taste in Drosophila. J Neurobiol 61: 333-342.

Hoffmann J. 2003. Anticipatory behavioural control. In Anticipatory behaviour in adaptive learning systems (ed. M Butz, et al.), pp. 44-65. Springer, Heidelberg.

Honjo K, Furukubo-Tokunaga K. 2005. Induction of cAMP response element-binding protein-dependent medium-term memory by appetitive gustatory reinforcement in Drosophila larvae. J Neurosci 25: 7905-7913.

Honjo K, Furukubo-Tokunaga K. 2009. Distinctive neuronal networks and biochemical pathways for appetitive and aversive memory in Drosophila larvae. J Neurosci 29: 852-862.

Ito K, Suzuki K, Estes P, Ramaswami M, Yamamoto D, Strausfeld NJ. 1998. The organization of extrinsic neurons and their implications in the functional roles of the mushroom bodies in Drosophila melanogaster Meigen. Learn Mem 5: 52-77.

Khurana S, Abu Baker MB, Siddiqi O. 2009. Odour avoidance learning in the larva of Drosophila melanogaster. J Biosci 34: 621-631.

Krashes MJ, DasGupta S, Vreede A, White B, Armstrong JD, Waddell S 2009. A neural circuit mechanism integrating motivational state with memory expression in Drosophila. Cell 139: 416-427.

Kreher SA, Kwon JY, Carlson JR. 2005. The molecular basis of odor coding in the Drosophila larva. Neuron 46: 445-456.

Kreher SA, Mathew D, Kim J, Carlson JR. 2008. Translation of sensory input into behavioral output via an olfactory system. Neuron 59: $110-124$.

Lee T, Lee A, Luo L. 1999. Development of the Drosophila mushroom bodies: Sequential generation of three distinct types of neurons from a neuroblast. Development 126: 4065-4076.
Liu L, Leonard AS, Motto DG, Feller MA, Price MP, Johnson WA, Welsh MJ. 2003. Contribution of Drosophila DEG/ENaC genes to salt taste. Neuron 39: $133-146$.

Marella S, Fischler W, Kong P, Asgarian S, Rueckert E, Scott K. 2006. Imaging taste responses in the fly brain reveals a functional map of taste category and behavior. Neuron 49: 285-295.

Marin EC, Watts RJ, Tanaka NK, Ito K, Luo L. 2005. Developmentally programmed remodeling of the Drosophila olfactory circuit. Development 132: 725-737.

Masuda-Nakagawa LM, Tanaka NK, O'Kane CJ. 2005. Stereotypic and random patterns of connectivity in the larval mushroom body calyx of Drosophila. Proc Natl Acad Sci 102: 19027-19032.

Masuda-Nakagawa LM, Gendre N, O'Kane CJ, Stocker RF. 2009. Localized olfactory representation in mushroom bodies of Drosophila larvae. Proc Natl Acad Sci 106: 10314-10319.

Matsumoto Y, Mizunami M. 2002. Temporal determinants of long-term retention of olfactory memory in the cricket Gryllus bimaculatus. J Exp Biol 205: 1429-1437.

McGuire SE, Le PT, Davis RL. 2001. The role of Drosophila mushroom body signaling in olfactory memory. Science 293: 1330-1333.

Melcher C, Pankratz MJ. 2005. Candidate gustatory interneurons modulating feeding behavior in the Drosophila brain. PLoS Biol 3: e305.

Menzel R. 2001. Searching for the memory trace in a mini-brain, the honeybee. Learn Mem 8: 53-62.

Meunier N, Marion-Poll F, Rospars JP, Tanimura T. 2003. Peripheral coding of bitter taste in Drosophila. J Neurobiol 56: 139-152.

Michels B, Chen YC, Saumweber T, Mishra D, Tanimoto H, Schmid B, Engmann O, Gerber B. 2011. Cellular site and molecular mode of synapsin action in associative learning. Learn Mem 18: $332-344$.

Miyakawa Y. 1982. Behavioral evidence for the existence of sugar, salt and amino acid recptor cells and some of their properties in Drosophila larvae. J Insect Physiol 28: 405-410.

Neuser K, Husse J, Stock P, Gerber B. 2005. Appetitive olfactory learning in Drosophila larvae: Effects of repetition, reward strength, age, gender, assay type and memory span. Anim Behav 69: 891-898.

Niewalda T, Singhal N, Fiala A, Saumweber T, Wegener S, Gerber B. 2008. Salt processing in larval Drosophila: Choice, feeding, and learning shift from appetitive to aversive in a concentration-dependent way. Chem Senses 33: 685-692.

Ofstad TA, Zuker CS, Reiser MB. 2011. Visual place learning in Drosophila melanogaster. Nature 474: 204-207.

Pauls D, Pfitzenmaier JE, Krebs-Wheaton R, Selcho M, Stocker RF, Thum AS. 2010. Electric shock-induced associative olfactory learning in Drosophila larvae. Chem Senses 35: 335-346.

Python F, Stocker RF. 2002. Adult-like complexity of the larval antennal lobe of $D$. melanogaster despite markedly low numbers of odorant receptor neurons. J Comp Neurol 445: 374-387.

Ramaekers A, Magnenat E, Marin EC, Gendre N, Jefferis GS, Luo L, Stocker RF. 2005. Glomerular maps without cellular redundancy at successive levels of the Drosophila larval olfactory circuit. Curr Biol 15: 982-992.

Riemensperger T, Voller T, Stock P, Buchner E, Fiala A. 2005. Punishment prediction by dopaminergic neurons in Drosophila. Curr Biol 15: 1953-1960.

Rodrigues V. 1980. Olfactory behavior of Drosophila melanogaster. In Development and neurobiology of Drosophila, Vol 16 (ed. O Siddiqi, et al.), pp. 361-371. Plenum, New York.

Salloum A, Colson V, Marion-Poll F. 2011. Appetitive and Aversive Learning in Spodoptera littoralis Larvae. Chem Senses. doi: 10.1093/chemse/ bjr041.

Saumweber T, Husse J, Gerber B. 2011. Innate attractiveness and associative learnability of odors can be dissociated in larval Drosophila. Chem Senses 36: $223-235$

Scherer S, Stocker RF, Gerber B. 2003. Olfactory learning in individually assayed Drosophila larvae. Learn Mem 10: 217-225.

Schipanski A, Yarali A, Niewalda T, Gerber B. 2008. Behavioral analyses of sugar processing in choice, feeding, and learning in larval Drosophila. Chem Senses 33: 563-573.

Schleyer M. 2009. Formation and expression of olfactory memory in fruit fly larvae: a behaviour-based model. Diploma thesis. Universität Würzburg.

Schnaitmann C, Vogt K, Triphan T, Tanimoto H. 2010. Appetitive and aversive visual learning in freely moving Drosophila. Front Behav Neurosci 4: 10

Schroll C, Riemensperger T, Bucher D, Ehmer J, Voller T, Erbguth K, Gerber B, Hendel T, Nagel G, Buchner E, et al. 2006. Light-induced activation of distinct modulatory neurons triggers appetitive or aversive learning in Drosophila larvae. Curr Biol 16: 1741-1747.

Schwaerzel M, Monastirioti M, Scholz H, Friggi-Grelin F, Birman S, Heisenberg M. 2003. Dopamine and octopamine differentiate between 
aversive and appetitive olfactory memories in Drosophila. J Neurosci 23: 10495-10502.

Séjourné J, Plaçais P-Y, Aso Y, Siwanowicz I, Trannoy S, Thoma V, Tedjakumala SR, Rubin GM, Tchénio P, Ito K, et al. 2011. Mushroom body efferent neurons responsible for aversive olfactory memory retrieval in Drosophila. Nat Neurosci 14: 903-910.

Selcho M, Pauls D, Han KA, Stocker RF, Thum AS. 2009. The role of dopamine in Drosophila larval classical olfactory conditioning. PLoS ONE 4: pe5897. doi: 10.1371/journal.pone.0005897.

Shiraiwa T. 2008. Multimodal chemosensory integration through the maxillary palp in Drosophila. PLoS ONE 3: pe2191. doi: 10.1371/ journal.pone.0002191.

Singh R, Singh K. 1984. Fine structure of the sensory organs of Drosophila melanogaster Meigen larva (Diptera: Drosophilidae). Int J Insect Morphol Embryol 13: 255-273.

Sokolowski MB. 2001. Drosophila: Genetics meets behaviour. Nat Rev Genet 2: $879-890$

Stocker RF. 2008. Design of the larval chemosensory system. In Brain development in Drosophila melanogaster (ed. GM Technau). Eureka-Landes Bioscience, Georgetown, TX.

Tanaka NK, Tanimoto H, Ito K. 2008. Neuronal assemblies of the Drosophila mushroom body. J Comp Neurol 508: 711-755.
Technau G, Heisenberg M. 1982. Neural reorganization during metamorphosis of the corpora pedunculata in Drosophila melanogaster. Nature 295: 405-407.

Tempel BL, Bonini N, Dawson DR, Quinn WG. 1983. Reward learning in normal and mutant Drosophila. Proc Natl Acad Sci 80: $1482-1486$.

Tomchik SM, Davis RL. 2009. Dynamics of learning-related cAMP signaling and stimulus integration in the Drosophila olfactory pathway. Neuron 64: $510-521$.

Tully T, Quinn WG. 1985. Classical conditioning and retention in normal and mutant Drosophila melanogaster. J Comp Physiol 157: 263-277.

Yarali A, Hendel T, Gerber B. 2006. Olfactory learning and behaviour are "insulated" against visual processing in larval Drosophila. J Comp Physiol A Neuroethol Sens Neural Behav Physiol 192: $1133-1145$.

Zhang K, Guo JZ, Peng Y, Xi W, Guo A. 2007. Dopamine-mushroom body circuit regulates saliency-based decision-making in Drosophila. Science 316: $1901-1904$

Received July 19, 2011; accepted in revised form July 26, 2011. 


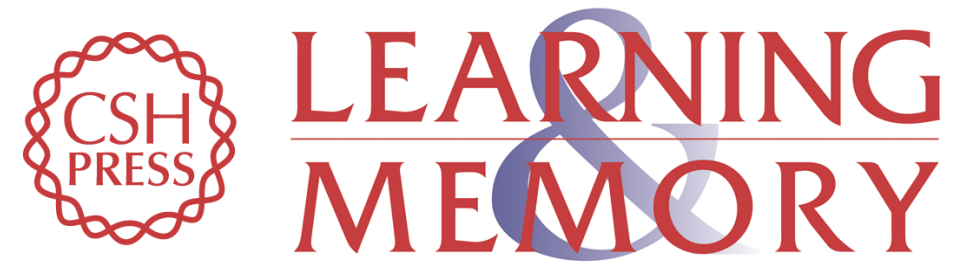

\section{A behavior-based circuit model of how outcome expectations organize learned behavior in larval Drosophila}

Michael Schleyer, Timo Saumweber, Wiebke Nahrendorf, et al.

Learn. Mem. 2011, 18:

Access the most recent version at doi:10.1101/lm.2163411 Supplemental
Material http://learnmem.cshlp.org/content/suppl/2011/09/26/18.10.639.DC1

References This article cites 77 articles, 22 of which can be accessed free at: http://learnmem.cshlp.org/content/18/10/639.full.html\#ref-list-1

License

Email Alerting Receive free email alerts when new articles cite this article - sign up in the box at the Service top right corner of the article or click here. 